\title{
PROJECTIVE SYSTEMIC MODULES
}

\author{
JAIUNG JUN, KALINA MINCHEVA, AND LOUIS ROWEN
}

\begin{abstract}
We develop the basic theory of projective modules and splitting in the more general setting of systems. Systems provide a common language for most tropical algebraic approaches including supertropical algebra, hyperrings (specifically hyperfields), and fuzzy rings. This enables us to prove analogues of classical theorems for tropical and hyperring theory in a unified way. In this context we prove a Dual Basis Lemma and versions of Schanuel's Lemma.
\end{abstract}

\section{INTRODUCTION}

\subsection{Motivation.}

In recent years, there has been a growing interest in developing theories of algebraic structures, more general than (commutative) rings, such as semirings, hyperrings (specifically hyperfields), fuzzy rings and supertropical algebra. The main motivation for the study of these structures is the number of applications to other areas of mathematics. For instance, semirings arise naturally in tropical algebraic geometry [34]. Using hyperfields (which generalize fields by allowing "multi-valued" addition) Baker and Bowler in 3 successfully unify various generalizations of matroids (combinatorial abstraction of vector spaces) in an elegant way. Baker and Bowler's work is based on the interesting idea (as initiated by the third author in 39 building on [15] and 31, and then [26]), that one is able to treat certain well-known (but not directly related) mathematical structures simultaneously. To this end, one needs to appeal to more general algebraic structures than commutative rings (cf. [5]).

The idea of proving results about classical objects, using these general algebraic structures, has already been implemented in the literature. For example, in [24] the first author proved that several topological spaces (algebraic varieties, Berkovich analytifications, and real schemes) can be seen as sets of " $H$ rational points" of algebraic varieties for some hyperfields $H$. In [2, Anderson and Davis defined the notion of hyperfield Grassmannians, generalizing a MacPhersonian (certain moduli space of matroids). Furthermore, in a recent paper 4, Baker and Lorscheid proved that certain moduli functors (of matroids) are representable by algebraic structures called pasteurized ordered blue prints.

In this paper, we continue developing the theory of the common framework for the generalized algebra structures, called a system. As indicated in Example 2.16, this "systemic" theory encompasses most algebraic approaches to tropical mathematics. In Examples 2.24 and 3.5 we state explicitly for the reader's convenience how the systemic (generalized) version of morphisms (which we call $\preceq$-morphisms) translates to tropical mathematics, hyperrings, and fuzzy rings.

In 21 the famous basic structure theorems about composition series, noetherian and artinian properties, etc., were studied for a specific class of modules, called SA-modules. These arise in tropical algebra, but not in classical algebra. Here we take a more categorical perspective, and projective modules play the major role. Projective modules over semirings, whose theory is analogous to classical exact sequences and module theory, appear in [17, Chapter 17] and [27, and have been studied rather intensively over the years, $10,19,20,28,29,35$.

An equivalent definition of projective module in classical ring theory is as a direct summand of a free module, but over an arbitrary semiring this property is considerably stronger. (See [20, Example 4.6] for a projective module over a semiring which is not a summand of a free module). The strong decomposition results given in 20, rely on this more restrictive definition and show that all indecomposable "strongly"

2010 Mathematics Subject Classification. Primary 16Y60, 13C10, 13C60, 20N20, 08A05; Secondary 06F05, 14T05, 08A72, 12K10, 16D40.

Key words and phrases. System, quasi-zero, triple, metatangible, negation map, symmetrization, congruence, tropical algebra, hypergroup, projective, dual basis, supertropical algebra, module, Schanuel's Lemma, semiring, surpassing relation. 
projective modules over a ring "lacking zero sums" are principal, and thus the Grothendieck group is trivial. This view is continued in [30]. However, direct sums are "too good" to lead to a viable homology theory over semirings.

In this paper we return to the general categorical definition of projective, cast in the language of systems, a crucial feature of which is the "surpassing relation" $\preceq$ which generalizes equality and is needed to reformulate analogs of classical ring-theoretic theorems in the semiring context. We consider more

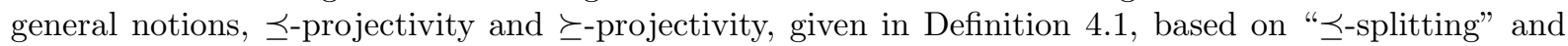
" $\succeq$-splitting" in Definition 3.10 . Bringing $\preceq$ into the picture, has the following advantages:

(i) $\preceq$-morphisms include tropicalization, as indicated in Example 2.24(i).

(ii) Idempotent mathematics can be formulated as a special case of the symmetrized semiring and module.

(iii) Applications include modules over hyperfields.

(iv) The class of projectives is broadened to include Example 4.19

(v) Classical results about projective modules (their characterization, the Dual Basis Lemma, and Schanuel's Lemma) are a special case of the $\preceq$-version, many of which are not available without the use of $\preceq$.

(vi) One can continue in analogy with classical lines, such as Morita theory, which already has been treated in 29] and [41. This thread is continued in homological algebra 25] and other work in progress.

Along the way, a more appropriate (and more general) $\preceq$-version and $\succeq$-version of direct sum is given in Definition 3.12 , via systemic generalization of splitting in $\$ 3.3$ and characterizations of $\preceq$-projectivity in 4.1 .

\subsection{Main results.}

Theorem A (Theorem 3.13). Let $\pi: \mathcal{M} \rightarrow \mathcal{N}$ be a homomorphism. If $\nu \preceq$-splits $\pi$, then:

(i) $\mathcal{M}$ is the $\preceq$-direct sum of $\mathcal{M}_{1}:=\pi(\mathcal{M})$ and $\mathcal{M}_{2}:=\left(\mathbb{1}_{\mathcal{M}}(-) \nu \pi\right)(\mathcal{M})$ with respect to the $\preceq$ morphisms $\pi_{1}=\pi, \nu_{1}=\nu, \pi_{2}=\left(\mathbb{1}_{\mathcal{M}}(-) \nu \pi\right), \nu_{2}=\mathbb{1}_{\mathcal{M}_{2}}$.

(ii) $\mathcal{M}$ is the $\preceq$-direct sum of $\mathcal{M}_{1}=\nu \pi(\mathcal{M})$ and $\mathcal{M}_{2}=\operatorname{ker}_{\mathrm{Mod}, \mathcal{M}} \pi$, with respect to $\nu_{i}=\mathbb{1}_{\mathcal{M}_{i}}$ for $i=1,2$.

This statement holds for $h$-splitting as well.

Proposition B (Proposition 4.4). The following are equivalent for a systemic module $\mathcal{P}$ :

(i) $\mathcal{P}$ is $(\preceq, h)$-projective.

(ii) Every $\preceq$-onto homomorphism to $\mathcal{P} \preceq$-splits.

(iii) There is a $\preceq$-onto homomorphism from a free system to $\mathcal{P}$ that $\preceq$-splits.

(iv) Given a $\preceq$-onto $\preceq$-morphism $h: \mathcal{M} \rightarrow \mathcal{M}^{\prime}$, the map $\operatorname{Mor}_{\preceq}(\mathcal{P}, h): \operatorname{Mor}_{\preceq}(\mathcal{P}, \mathcal{M}) \rightarrow \operatorname{Mor}_{\preceq}\left(\mathcal{P}, \mathcal{M}^{\prime}\right)$ given by $g \mapsto h g$ is $\preceq$-onto.

The h-version also holds (Proposition 4.6).

Proposition B' (Proposition 4.7). The following are equivalent for a systemic module $\mathcal{P}$ :

(i) $\mathcal{P}$ is $\succeq$-projective.

(ii) Every $\succeq$-onto $\preceq$-morphism to $\mathcal{P} \succeq$-splits.

(iii) There is a $\succeq$-onto $\preceq$-morphism from a free system to $\mathcal{P}$ that $h$ - $\succeq$-splits.

(iv) The functor $\operatorname{Hom}\left(\mathcal{P}, \_\right)$sends $\succeq$-onto $\preceq$-morphisms to $\succeq$-onto $\preceq$-morphisms.

In the following statements we use various notions of kernel for a map $f: \mathcal{M} \rightarrow \mathcal{N}$ (cf. Definition 3.1 and Definition 5.1):

(1) Null-module kernel $\operatorname{ker}_{\text {Mod, } \mathcal{M}} f$ defined as the preimage of the set $\{a \in \mathcal{N}: a \succeq \mathbb{O}\}$.

(2) Congruence kernel $\operatorname{ker}_{N} f:=\left\{\left(a_{0}, a_{1}\right) \in \mathcal{M} \times \mathcal{M}: f\left(a_{0}\right)=f\left(a_{1}\right)\right\}$.

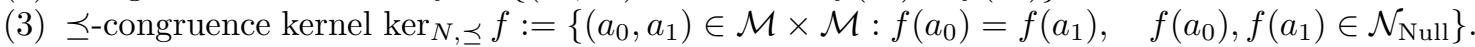

Theorem C (Theorem 4.15). If $\mathcal{P}_{1}$ is $(\preceq, h)$-projective with a $\preceq$-onto homomorphism $\pi: \mathcal{P} \longrightarrow \mathcal{P}_{1}$ whose null-module kernel $\mathcal{K}$ is $(\preceq, h)$-projective, then $\mathcal{P}$ also is $(\preceq, h)$-projective. 
Proposition D (Proposition 4.21_ “ $\preceq$-Dual Basis Lemma"). A module pseudo-system $\left(\mathcal{P}, \mathcal{T}_{\mathcal{P}},(-), \preceq\right)$ that is $\preceq$-generated by $\left\{p_{i} \in \mathcal{P}: i \in I\right\}$ is $(\preceq, h)$-projective (resp. h-projective) if and only if there are $\preceq$-onto $\preceq$-morphisms (resp. homomorphisms) $g_{i}: \mathcal{P} \rightarrow \mathcal{A}$ such that for all $a \in \mathcal{A}$ we have $a \preceq \sum g_{i}(a) p_{i}$, where $g_{i}(a)=\mathbb{0}$ for all but finitely many $i$.

Proposition D' (Proposition4.22_ “ $\succeq$-Dual Basis Lemma”). Suppose a module pseudo-system $\left(\mathcal{P}, \mathcal{T}_{\mathcal{P}},(-), \preceq\right.$ ) is generated by $\left\{p_{i} \in \mathcal{P}: i \in I\right\}$. Then $\mathcal{P}$ is $(\succeq, h)$-projective if and only if there are $\succeq$-onto $\succeq$-morphisms $g_{i}: \mathcal{P} \rightarrow \mathcal{A}$ such that for all $a \in \mathcal{A}$ we have $a \succeq \sum g_{i}(a) p_{i}$, where $g_{i}(a)=\mathbb{O}$ for all but finitely many $i$.

These are tied in with $\preceq$-idempotent and $\preceq$-von Neumann regular matrices in Proposition 4.17 and Corollary 4.18 .

With the basic definitions and properties in hand, one is ready to embark on the part of module theory involving projective modules. Our main application is Schanuel's Lemma over semirings.

Theorem E (Theorem [5.3). Suppose we have $\preceq$-morphisms $\mathcal{P}_{1} \stackrel{f_{1}}{\longrightarrow} \mathcal{M}$ and $\mathcal{P}_{2} \stackrel{f_{2}}{\longrightarrow} \mathcal{M}$ with $f_{1}$ and $f_{2}$ onto. (We are not assuming that either $\mathcal{P}_{i}$ is projective.) Let

$$
\mathcal{P}=\left\{\left(b_{1}, b_{2}\right): \quad b_{i} \in \mathcal{P}_{i}, f_{1}\left(b_{1}\right)=f_{2}\left(b_{2}\right)\right\},
$$

a submodule of $\mathcal{P}_{1} \oplus \mathcal{P}_{2}$, together the restriction $\pi_{i}^{\text {res }}$ of the projection $\pi_{i}: \mathcal{P} \rightarrow \mathcal{P}_{i}$ on the $i$ coordinate, for $i=1,2$.

(i) $\pi_{1}^{\text {res }}: \mathcal{P} \rightarrow \mathcal{P}_{1}$ is an onto homomorphism and and there is an onto homomorphism

$$
\operatorname{ker}_{N} \pi_{1}^{\mathrm{res}} \rightarrow \operatorname{ker}_{N} f_{2},
$$

(This part is purely semiring-theoretic and does not require a system.)

(ii) The maps $f_{1} \pi_{1}^{\text {res }}, f_{2} \pi_{2}^{\text {res }}: \mathcal{P} \rightarrow \mathcal{M}$ are the same.

(iii) In the systemic setting, $\pi_{1}^{\text {res }}$ also induces $\preceq$-quasi-isomorphism

$$
\pi_{N, \preceq}: \operatorname{ker}_{N, \preceq} \pi_{1}^{\text {res }} \rightarrow \operatorname{ker}_{N, \preceq} f_{2} .
$$

(iv) In (iii), if $f_{1}$ also is null-monic, we have the following $\preceq$-quasi-isomorphism:

$$
\operatorname{ker}_{N, \preceq} f_{1} \pi_{1}^{\text {res }} \rightarrow \operatorname{ker}_{N, \preceq} f_{2} .
$$

(v) If $\mathcal{P}_{1}$ is projective, then it is a retract of $\mathcal{P}$ with respect to the projection $\pi_{1}: \mathcal{P} \rightarrow \mathcal{P}_{1}$.

(vi) If $\mathcal{P}_{1}$ is $\preceq$-projective, then it is a $\preceq$-retract of $\mathcal{P}$ with respect to the projection $\pi_{1}: \mathcal{P} \rightarrow \mathcal{P}_{1}$, and $\mathcal{P}$ is the $\preceq$-direct sum of $\mathcal{P}_{1}$ and $\left(\mathbb{1}_{\mathcal{P}}(-) \nu_{1} \pi_{1}\right)(\mathcal{P})$.

We also have a $\preceq$-onto $\preceq$-version, given in Lemma 5.5 .

Theorem F (Theorem 5.6 (Semi-Schanuel, $\preceq$-version)). Given $a \preceq$-morphism $\mathcal{P} \stackrel{f}{\longrightarrow} \mathcal{M}$ and a homomorphism $\mathcal{P}^{\prime} \stackrel{f^{\prime}}{\longrightarrow} \mathcal{M}^{\prime}$ with $\mathcal{P}$ and $\mathcal{P}^{\prime} \preceq$-projective and $f \preceq$-onto, and $a \preceq$-onto $\preceq$-morphism $\mu: \mathcal{M} \rightarrow \mathcal{M}^{\prime}$, let $\mathcal{K}=\operatorname{ker}_{\text {Mod, } \mathcal{P}} f$ and $\mathcal{K}^{\prime}=\operatorname{ker}_{\text {Mod, }} \mathcal{P}^{\prime} f^{\prime}$. Then there is a $\preceq$-onto $\preceq$-splitting $\preceq$-morphism $g: \mathcal{K}^{\prime} \oplus \mathcal{P} \rightarrow \mathcal{P}^{\prime}$, with a $\preceq$-morphism $\Phi: \mathcal{K} \rightarrow \operatorname{ker}_{\mathrm{Mod}, \mathcal{K}^{\prime} \oplus \mathcal{P}} g$ which is 1:1 (as a set-map).

This could be used in conjunction with Theorem 4.15

Our approach throughout this paper is explicit, aimed to show how projective systemic modules work, especially since subtle variations of the definitions lead to differing results. The category of $\mathcal{T}$-modules has enough $(\preceq, h)$-projectives and $h$-projectives (because every free module is also projective, $(\preceq, h)$ projective, and h-projective) to define $\preceq$-projective resolutions, but despite Theorem 4.15 we do not yet have decisive enough results along these lines to include here (although Corollary 5.7 indicates how the theory might develop).

Acknowledgments J.J. was supported by AMS-Simons travel grant. K.M. was supported by the Institute Mittag-Leffler and the "Vergstiftelsen". K.M. would like to thank the Institute Mittag-Leffler for its hospitality. Part of this work has been carried out during the workshop "Workshop on Tropical varieties and amoebas in higher dimension" in which K.M and L.R. participated. 


\section{BASIC NOTIONS}

Throughout the paper, we let $\mathbb{N}$ be the additive monoid of the non-negative integers. Similarly, we view $\mathbb{Q}$ (resp. $\mathbb{R}$ ) as the additive monoid of the rational numbers (resp. of the real numbers).

A semiring $(\mathcal{A},+, \cdot, 1)$ is an additive commutative monoid $(\mathcal{A},+, \mathbb{0})$ and multiplicative monoid $(\mathcal{A}, \cdot, \mathbb{1})$ satisfying the usual distributive laws, cf. [17.

Remark 2.1. Strictly speaking the element $\mathbb{O}$ is not needed in semiring theory, and one can make do later by adjoining the absorbing element $\mathbb{0}$, but for convenience we will work with semirings and assume $\mathbb{0} \in \mathcal{A}$.

We review the basic definitions and properties of $\mathcal{T}$-modules, triples, and systems from [40; more details are given in [26] and [39.

Definition 2.2. $A \mathcal{T}$-module over a set $\mathcal{T}$ is an additive monoid $\left(\mathcal{A},+, \mathbb{D}_{\mathcal{A}}\right)$ with a scalar multiplication $\mathcal{T} \times \mathcal{A} \rightarrow \mathcal{A}$ satisfying the following axioms, $\forall u \in \mathbb{N}, a \in \mathcal{T}, b, b_{j} \in \mathcal{A}$ :

(i) (Distributivity over $\mathcal{T}): a\left(\sum_{j=1}^{u} b_{j}\right)=\sum_{j=1}^{u}\left(a b_{j}\right)$.

(ii) $a \mathbb{D}_{\mathcal{A}}=\mathbb{D}_{\mathcal{A}}$.

We review some definitions for convenience. We start off with a $\mathcal{T}$-module $\mathcal{A}$, perhaps with extra structure. When $\mathcal{T}$ is a monoid we call $\mathcal{A}$ a $\mathcal{T}$-monoid module. We can make $\mathcal{A}$ into a semiring by means of [39, Theorem 2.5], in which case we essentially have Lorscheid's blueprints, [31, 32.

2.1. Negation maps. We introduce some more structure.

Definition 2.3. A negation map on a $\mathcal{T}$-module $\mathcal{A}$ is a monoid isomorphism $(-): \mathcal{A} \rightarrow \mathcal{A}$ of order $\leq 2$, written $a \mapsto(-) a$, which also respects the $\mathcal{T}$-action in the sense that

$$
(-)(a b)=a((-) b)
$$

for $a \in \mathcal{T}, b \in \mathcal{A}$.

Assortments of negation maps are given in [14, 26, 39]. We also remark that when $\mathbb{1} \in \mathcal{T} \subseteq \mathcal{A}$, the negation map $(-)$ is given by $(-) b=((-) \mathbb{1}) b$ for $b \in \mathcal{A}$.

We write $a(-) a$ for $a+((-) a)$, and $a^{\circ}$ for $a(-) a$, called a quasi-zero.

Remark 2.4. Any quasi-zero is fixed by a negation map since $(-) a^{\circ}=(-) a+a=a^{\circ}$. On the other hand, when $\mathcal{A}$ is idempotent (i.e., $a+a=a$ for any $a \in \mathcal{A}$ ), any element $a \in \mathcal{A}$ fixed by a negation map is a quasi-zero since $a=(-) a$ and hence $a^{\circ}=a(-) a=a+a=a$.

The set $\mathcal{A}^{\circ}$ of quasi-zeros is a $\mathcal{T}$-submodule of $\mathcal{A}$ that plays an important role. When $\mathcal{A}$ is a semiring, $\mathcal{A}^{\circ}$ is an ideal.

Definition 2.5. A pseudo-triple $(\mathcal{A}, \mathcal{T},(-))$ is a $\mathcal{T}$-module $\mathcal{A}$, with $\mathcal{T}$ a distinguished subset of $\mathcal{A}$, called the set of tangible elements, and a negation map $(-)$ satisfying $(-) \mathcal{T}=\mathcal{T}$.

In this paper, we replace $\mathcal{T}$ by a subset $\mathcal{T}_{\mathcal{A}} \subseteq \mathcal{A}$. We write $\mathcal{T}_{\mathbb{D}}$ for $\mathcal{T} \cup\{\mathbb{0}\}$.

Definition 2.6. A triple $(\mathcal{A}, \mathcal{T},(-))$ is a pseudo-triple, for which $\mathcal{T} \cap \mathcal{A}^{\circ}=\emptyset$ and $\mathcal{T}_{\mathbb{0}}$ generates $(\mathcal{A},+)$.

\subsection{Symmetrization and idempotent mathematics.}

When a $\mathcal{T}$-module $\mathcal{A}$ does not come equipped with a negation map, there are two natural ways to impose a negation map: (1) one may take the negation (-) to be the identity (for instance, this is done in supertropical algebra), or (2) one may supply a negation map by "symmetrizing" $\mathcal{A}$, in a procedure similar to the Grothendieck group completion. For more details, see [15] and then [26, §1.3]. Symmetrization is an important tool for idempotent mathematics and the max-plus algebra, and plays a central role in our subsequent work [25]. We briefly recall the basic definitions for the reader.

For any $\mathcal{T}$-module $\mathcal{A}$, we let $\widehat{\mathcal{A}}=\mathcal{A} \oplus \mathcal{A}$ and $\widehat{\mathcal{T}}=(\mathcal{T} \oplus \mathbb{O}) \cup(\mathbb{D} \oplus \mathcal{T})$. The main idea, as in the case of the group completion, is to consider the formal construction of negation, and impose a canonical $\widehat{\mathcal{T}}$-module structure on $\widehat{\mathcal{A}}$ as follows. 
Definition 2.7. The twist action on $\widehat{\mathcal{A}}$ over $\widehat{\mathcal{T}}$ is defined as follows:

$$
\left(a_{0}, a_{1}\right) \cdot{ }_{\mathrm{tw}}\left(b_{0}, b_{1}\right)=\left(a_{0} b_{0}+a_{1} b_{1}, a_{0} b_{1}+a_{1} b_{0}\right), a_{i} \in \mathcal{T}, b_{i} \in \mathcal{A} .
$$

The symmetrization of $\mathcal{A}$ is the $\widehat{\mathcal{T}}$-module $\widehat{\mathcal{A}}$ with the twist action (2.1). A negation map is defined by using the switch map:

$$
(-): \widehat{\mathcal{A}} \longrightarrow \widehat{\mathcal{A}}, \quad\left(b_{0}, b_{1}\right) \mapsto\left(b_{1}, b_{0}\right) .
$$

When $\mathcal{A}$ is a semiring, the twist action gives a semiring structure on $\widehat{\mathcal{A}}$ (together with coordinate-wise addition).

Remark 2.8. The switch map $(-)$ on $\widehat{\mathcal{A}}$ is a negation map, and the quasi-zeros all have the form $(b, b)$ since $\left(b_{0}, b_{1}\right)(-)\left(b_{0}, b_{1}\right)=\left(b_{0}+b_{1}, b_{0}+b_{1}\right)$.

Suppose now that $\mathcal{T} \subseteq \mathcal{A}$. (One could even take $\mathcal{T}=\mathcal{A} \backslash \mathbb{O}$.) Then $\mathcal{T}_{\widehat{\mathcal{A}}} \cap \widehat{\mathcal{A}}^{\circ}=\emptyset$, and $\left(\widehat{\mathcal{A}}, \mathcal{T}_{\widehat{\mathcal{A}}},(-)\right)$ is a triple for any $\mathcal{T}$-module $(\mathcal{A}, \mathcal{T},(-))$.

The $\operatorname{map}(\mathcal{A}, \mathcal{T},(-)) \rightarrow(\widehat{\mathcal{A}}, \widehat{\mathcal{T}},(-))$ sending $a \mapsto(a, \mathbb{O})$ and $b \mapsto(b, \mathbb{O})$ for $a \in \mathcal{T}, b \in \mathcal{A}$, is a homomorphism of triples. When $\mathcal{A}$ is additively idempotent, so is $\widehat{\mathcal{A}}$. In this way, we embed idempotent mathematics into the theory of triples.

Thinking of $\left(b_{0}, b_{1}\right)$ intuitively as $b_{0}-b_{1}$, we see that $\left(b_{1}, b_{0}\right)$ corresponds to $b_{1}-b_{0}=-\left(b_{0}-b_{1}\right)$.

\subsection{Systems.}

We round out the structure with a surpassing relation $\preceq$ given in [39, Definition 1.70] and also described in [26, Definition 3.11].

Definition 2.9. A surpassing relation on a triple $(\mathcal{A}, \mathcal{T},(-))$, denoted $\preceq$, is a partial pre-order satisfying the following, for elements of $\mathcal{A}$ :

(i) $c^{\circ} \succeq \mathbb{O}$ for any $c \in \mathcal{A}$.

(ii) If $b_{1} \preceq b_{2}$ then $(-) b_{1} \preceq(-) b_{2}$.

(iii) If $b_{1} \preceq b_{2}$ and $b_{1}^{\prime} \preceq b_{2}^{\prime}$ for $i=1,2$ then $b_{1}+b_{1}^{\prime} \preceq b_{2}+b_{2}^{\prime}$.

(iv) If $a \in \mathcal{T}$ and $b_{1} \preceq b_{2}$ then $a b_{1} \preceq a b_{2}$.

(v) If $a \preceq b$ for $a, b \in \mathcal{T}$, then $a=b$.

$A \mathcal{T}$-surpassing relation on a triple $\mathcal{A}$ is a surpassing relation also satisfying the following, for elements of $\mathcal{A}:$ if $b \preceq a$ for $a \in \mathcal{T}$ and $b \in \mathcal{A}$, then $b=a$.

Lemma 2.10. If $b_{1}+c^{\circ}=b$ for some $c \in \mathcal{A}$, then $b_{1} \preceq b$.

Proof. Since $c^{\circ} \succeq \mathbb{0}$, we can apply Definition 2.9 (iii).

The main case is in [39, Definition 1.70], [26, Definition 2.17], defined as follows:

$$
a_{1} \preceq_{\circ} a_{2} \text { if } a_{2}=a_{1}+b^{\circ} \text { for some } b \in \mathcal{A},
$$

but we also could take $\preceq$ to be set inclusion when $\mathcal{A}$ is obtained from the power set of a hyperring. See [26, $§ 10]$.

Lemma 2.11. If $a_{1} \preceq a_{2}$, then $a_{2}(-) a_{1} \succeq \mathbb{O}$ and $a_{1}(-) a_{2} \succeq \mathbb{0}$.

Proof. $a_{2}(-) a_{1} \succeq a_{1}(-) a_{1} \succeq \mathbb{0}$, and thus $a_{1}(-) a_{2}=(-)\left(a_{2}(-) a_{1}\right) \succeq \mathbb{0}$.

Definition 2.12. $S_{1} \preceq S_{2}$ for $S_{1}, S_{2} \subseteq \mathcal{A}$ if for each $s \in S_{1}$ there is $s^{\prime} \in S_{2}$ for which $s \preceq s^{\prime}$.

Definition 2.13. A system (resp. pseudo-system) is a quadruple $(\mathcal{A}, \mathcal{T},(-), \preceq)$, where $\preceq$ is a surpassing relation on the triple (resp. pseudo-triple) $\left(\mathcal{A}, \mathcal{T}_{\mathcal{A}},(-)\right)$, which is uniquely negated in the sense that for any $a \in \mathcal{T}$, there is a unique element $b$ of $\mathcal{T}_{\mathcal{A}}$ for which $\mathbb{0} \preceq a+b$ (namely $\left.b=(-) a\right)$.

$A \mathcal{T}$-system is a system for which $\preceq$ is a $\mathcal{T}$-surpassing relation.

Remark 2.14. Pseudo-systems encompass classical algebra, when we take (-) to be the usual negation - , and $\preceq$ to be equality. This "explains" the parallel between so many theorems of tropical algebra and classical algebra.

For a pseudo-system $(\mathcal{A}, \mathcal{T},(-), \preceq)$, we define the important $\mathcal{T}$-submodule $\mathcal{A}_{\mathrm{Null}}=\{a \in \mathcal{A}: a \succeq \mathbb{O}\}$ of $\mathcal{A}$ containing $\mathcal{A}^{\circ}$.

Then in parallel to (2.2) we have 
Definition 2.15. $b \preceq_{\mathrm{Null}} b^{\prime}$ when $b+c=b^{\prime}$ for some $c \in \mathcal{A}_{\mathrm{Null}}$.

There are two ways that we want to view triples and their systems. The first is as the ground structure on which we build our module theory, in analogy to the ground ring for classical linear algebra or for affine algebraic geometry. We call this a ground system. We call $\mathcal{A}$ a semiring system when $\mathcal{A}$ is a semiring.

The second way, which is the main direction taken in this paper, is to fix a ground triple $(\mathcal{A}, \mathcal{T},(-))$, and take $\mathcal{A}$-modules $\mathcal{M}$ together with a distinguished subset $\mathcal{T}_{\mathcal{M}}$ spanning $\mathcal{M}$ and satisfying $\mathcal{T}_{\mathcal{M}} \subseteq \mathcal{T}_{\mathcal{M}}$. We also require $\mathcal{M}$ to satisfy $((-) a) m=(-)(a m)$ for $a \in \mathcal{A}, m \in \mathcal{M}$. Then we define the systemic module $\left(\mathcal{M}, \mathcal{T}_{\mathcal{M}},(-), \preceq\right)$ on $\mathcal{M}$, to satisfy the axiom

$a_{1} b_{1} \preceq a_{2} b_{2}$ whenever $a_{1} \preceq a_{2}$ in $\mathcal{T}_{\mathcal{M}}$ and $b_{1} \preceq b_{2}$ in $\mathcal{M}$.

Right systemic modules are defined analogously. The detailed study of such modules was carried out in [26]. Although the two theories (ground systems and systemic modules) start off the same, they quickly diverge, just as in classical algebra.

\section{Example 2.16.}

(i) Given a triple $(\mathcal{A}, \mathcal{T},(-))$, take the surpassing relation $\preceq$ to be $\preceq$ of $(2.2)$; then $\mathcal{A}_{\mathrm{Null}}=\mathcal{A}^{\circ}$.

(ii) The set-up of supertropical mathematics [18, 22] is a special case of (i), where $\mathcal{A}=\mathcal{T} \cup \mathcal{G}$ is the supertropical semiring, (-) is the identity, ○ is the "ghost map," $\mathcal{G}=\mathcal{A}^{\circ}$, and $\preceq$ is "ghost surpasses". Another way of saying this is that $a_{0}+a_{1} \in\left\{a_{0}, a_{1}\right\}$ for $a_{0} \neq a_{1} \in \mathcal{T}$, and $a_{0}+a_{0}=a_{0}^{\circ}$. Tropical mathematics is encoded in $\mathcal{G}$, which (excluding $\mathbb{0}$ ) often is an ordered group, and can be viewed for example as the target of the Puiseux valuation (tropicalization).

(iii) The fuzzy ring of [11] is a special case of (i). More details are given in [39] and [1].

(iv) The symmetrized triple can be made into a system as special case of (i), which includes idempotent mathematics, as was explained in Remark 2.8 .

(v) In the hypergroup setting, as described in [39, Definition 3.47], $\mathcal{T}$ is a given hypergroup, $\mathcal{A}$ is the subset of the power set $\mathcal{P}(\mathcal{T})$ generated by $\mathcal{T}$, and $\preceq$ is set inclusion. We call this a hypersystem. $\mathcal{A}_{\mathrm{Null}}$ consists of those sets containing $\mathbb{0}$, which is the set of hyperzeros in the hypergroup literature.

(vi) Tracts, introduced recently in [3], are mostly special cases of systems, where $\mathcal{T}$ is the given Abelian group $G, \mathcal{A}=\mathbb{N}[G], \varepsilon=(-) \mathbb{1}$, and $N_{G}$ is $\mathcal{A}_{\mathrm{Null}}$, usually taken to be $\mathcal{A}^{\circ}$.

Examples 2.16 can be unified for systems by taking $\preceq$ to be $\preceq_{\text {Null }}$ of Definition 2.15. Clearly this includes Example 2.16(i), and it also includes Example 2.16(v) since $c \in \mathcal{A}_{\mathrm{Null}}$ iff $\mathbb{0} \in c$, which implies $b \subseteq b+c$, for $b, c \subseteq \mathcal{P}(\mathcal{T})$. We will also want a weaker version of generation, which comes up naturally and also ties into hyperrings.

Remark 2.17. In a semiring, one has the Green relation given by $a \leq b$ iff $a+b=b$, 39, Example 2.60(i)]. Conversely, any ordered monoid with $\mathbb{0}$ gives rise to an idempotent semiring by putting $a+b=b$ whenever $a \leq b$.

The only natural negation map here would be the identity, and one gets a pseudo-triple by taking $\mathcal{T}$ to be a generating set of $\mathcal{A}$. But every element $a=a+a$ is a quasi-zero, and $\mathcal{A}_{\mathrm{Null}}=\mathcal{A}$, so this pseudosystem is not a system, and one does not get much structure theory along the lines of systems. This is remedied in Example 2.16(ii), by symmetrization.

In the spirit of systems, our semirings will rather be "almost" idempotent (specifically "(-)-bipotent," cf. [39, Definition 2.27] and [26, Definition 2.8]).

Lemma 2.18. Hypersystems $(\mathcal{A}=\mathcal{P}(\mathcal{T}), \mathcal{T},(-), \subseteq)$ satisfy the following property:

If $a \in \mathcal{T}$ and $a+b \succeq \mathbb{0}$ for $b \in \mathcal{A}$, then $(-) a \preceq b$.

Proof. $\mathbb{0} \preceq a+b$ means that there exists $q \in b$ such that $\mathbb{O} \in a+q$ (as a hypergroup). In particular, $q=-a$ and hence $(-a) \in b$, or $(-a) \preceq b$ since $\preceq$ is just the set-inclusion $\subseteq$ in this case.

Definition 2.19. Let $(\mathcal{A}, \mathcal{T},(-), \preceq)$ be a pseudo-system.

(i) An element $b \in \mathcal{A}$ is $\preceq$-generated by a subset $\mathcal{A}^{\prime}$ of $\mathcal{A}$ if there is a subset $\left\{a_{i}: 1 \leq i \leq t\right\} \subseteq \mathcal{A}^{\prime}$ such that $b \preceq \sum_{i} a_{i}$. 
(ii) For subsets $\mathcal{A}^{\prime}$ and $\mathcal{A}^{\prime \prime}$ of $\mathcal{A}$, we say that $\mathcal{A}^{\prime} \preceq$-generates $\mathcal{A}^{\prime \prime}$ if each element of $\mathcal{A}^{\prime \prime}$ is $\preceq$-generated by $\mathcal{A}^{\prime}$.

The $\succeq$-analog is less interesting because of the following reduction to usual generation.

Lemma 2.20. In a $\mathcal{T}$-system $\mathcal{A}$, with $\mathcal{A}^{\prime} \subseteq \mathcal{A}$, if for each $b \in \mathcal{A}$ there is $S_{b}=\left\{a_{i}: 1 \leq i \leq t\right\} \subseteq \mathcal{A}^{\prime}$ such that $b \succeq \sum_{i} a_{i}$, then $\mathcal{A}^{\prime}$ generates $\mathcal{A}$ in the usual sense.

Proof. For $b \in \mathcal{A}$, write $b=\sum a_{i}$, where $a_{i} \in \mathcal{T}$, and $\sum_{j} b_{i, j} \preceq a_{i}$ for $b_{i, j} \in \mathcal{A}^{\prime}$, implying $\sum_{j} b_{i, j}=a_{i}$ by Definition 2.9 for $\mathcal{T}$-systems, and thus $b=\sum_{i} \sum_{j} b_{i, j}$.

Definition 2.21. Let $\mathcal{A}$ be a system. $A \preceq$-morphism of $\mathcal{A}$-module pseudo-systems

$$
\varphi:\left(\mathcal{M}, \mathcal{T}_{\mathcal{M}},(-), \preceq\right) \rightarrow\left(\mathcal{M}^{\prime}, \mathcal{T}_{\mathcal{M}^{\prime}}^{\prime},(-)^{\prime}, \preceq^{\prime}\right)
$$

is a map $\varphi: \mathcal{M} \rightarrow \mathcal{M}^{\prime}$ satisfying the following properties for $a_{i} \in \mathcal{T}$ and $b \preceq b^{\prime}$ in $\mathcal{M}, b_{i}$ in $\mathcal{M}$ :

(i) $\varphi(\mathbb{O})=\mathbb{0}$.

(ii) $\varphi\left((-) b_{1}\right)=(-) \varphi\left(b_{1}\right)$;

(iii) $\varphi\left(b_{1}+b_{2}\right) \preceq^{\prime} \varphi\left(b_{1}\right)+\varphi\left(b_{2}\right)$;

(iv) $\varphi\left(a_{1} b\right)=a_{1} \varphi(b)$.

(v) $\varphi(b) \preceq \preceq^{\prime} \varphi\left(b^{\prime}\right)$.

By a homomorphism we mean the usual universal algebra definition, i.e., in (iii), equality holds instead of $\preceq$.

In many cases (such as for hypersystems) we also want to include the condition $\varphi(\mathcal{T}) \subseteq \mathcal{T}^{\prime}$, but there are instances for which we do not want this condition to hold (for example the zero morphism $a \mapsto \mathbb{D}$, the morphism $a \mapsto a^{\circ}$, or more generally, null morphisms of Definition 3.1 below).

\section{Remark 2.22.}

(i) $\varphi\left(\mathcal{M}_{\text {Null }}\right) \subseteq \mathcal{M}^{\prime}$ Null follows from conditions (i) and $(v)$, since $\mathbb{O} \preceq b$ implies $\mathbb{O}=\varphi(\mathbb{O}) \preceq^{\prime} \varphi(b)$.

(ii) To show condition (ii), it is enough to have $\varphi\left((-) a_{1}\right) \preceq^{\prime}(-) \varphi\left(a_{1}\right)$, by [26, Proposition 2.41].

(iii) $\varphi(b(-) c) \succeq \varphi(b)$, for all $c \succeq \mathbb{0}$, in view of (v) and Lemma 2.10 .

(iv) There also is a subtle issue concerning $\preceq$-morphisms of systemic modules; we would want $\varphi(\mathcal{T}) \cap$ $\mathcal{T}_{\mathcal{M}^{\prime}}$ to generate $\left(\mathcal{M}^{\prime},+\right)$, in order for $\varphi(\mathcal{M})$ to be a systemic module.

Lemma 2.23. When $\preceq^{\prime}$ is a PO (partial order) and $\mathcal{T}$ is a group, Definition 2.21(iv) is implied by the formally weaker condition

$$
\varphi(a b) \preceq^{\prime} a \varphi(b), \quad \forall a \in \mathcal{T} .
$$

Proof. $a \varphi(b)=a \varphi\left(a^{-1} a b\right) \preceq^{\prime} a a^{-1} \varphi(a b) \preceq^{\prime} a \varphi(b)$, so equality holds at each stage.

Analogously, by a $\succeq$-morphism we use the same definition as $\preceq$-morphism, except with (iii) now reading

$$
\varphi\left(b_{1}+b_{2}\right) \succeq^{\prime} \varphi\left(b_{1}\right)+\varphi\left(b_{2}\right) .
$$

Example 2.24. Let us describe these notions for Example 2.16; in the process we see why we want to consider $\preceq$-morphisms rather than just homomorphisms.

(i) In supertropical mathematics, a -morphism $f$ satisfies

$$
f\left(b_{1}+b_{2}\right)+\text { ghost }=f\left(b_{1}\right)+f\left(b_{2}\right)
$$

(2.3) implies that either $f\left(b_{1}+b_{2}\right)=f\left(b_{1}\right)+f\left(b_{2}\right)$, or $f\left(b_{1}\right)+f\left(b_{2}\right)$ is ghost, in which case either $f\left(b_{1}\right)=f\left(b_{2}\right)$, or $f\left(b_{1}\right)$ is ghost of value greater than or equal to $f\left(b_{2}\right)$ (or visa versa). In particular, this is the case for tropicalization of the Puiseux series via the Puiseux valuation, and is one of our main motivations in introducing $\preceq$-morphisms.

(ii) For hypersystems, a $\preceq$-morphism $f$ satisfies

$$
f\left(b_{1} \boxplus b_{2}\right) \subseteq f\left(b_{1}\right) \boxplus f\left(b_{2}\right),
$$

the definition used in [7, Definition 2.1] and [16, Definition 2.4]. This is intuitive when $f$ maps the hyperring $\mathcal{T}$ into itself. On the other hand, hyperring $\succeq$-morphisms which are not homomorphisms 
seem to be artificial; for an example, one could extend the identity on the phase hyperfield to a map that doubles all non-singleton arcs around the center.

Given a hypersystem $(\mathcal{A}=\mathcal{P}(\mathcal{T}), \mathcal{T},(-), \subseteq)$ and a hypergroup morphism $f$ over $\mathcal{T}$, it is natural to extend $f$ to $\mathcal{A}$ via

$$
f\left(\left\{a_{i}: a_{i} \in \mathcal{T}\right\}\right)=\left\{f\left(a_{i}\right): a_{i} \in \mathcal{T}\right\} .
$$

In this case, if $f(b)(-) f\left(b^{\prime}\right) \succeq \mathbb{0}$, there is some hypergroup element $a \in f(b) \cap f\left(b^{\prime}\right)$.

(iii) For fuzzy rings, in [11, § 1], also see [16, Definition 2.17 ], a homomorphism

$$
f:\left(K ;+; \times, \varepsilon_{K}, K_{0}\right) \rightarrow\left(L ;+; \times ; \varepsilon_{L} ; L_{0}\right)
$$

of fuzzy rings is defined as satisfying: For any $\left\{a_{1}, \ldots, a_{n}\right\} \in K^{\times}$if $\sum_{i=1}^{n} a_{i} \in K_{0}$ then $\sum_{i=1}^{n} f\left(a_{i}\right) \in L_{0}$. Any $\preceq$-morphism in our setting is a fuzzy homomorphism since $L_{0}$ is an ideal, and thus $\sum_{i=1}^{n} f\left(a_{i}\right) \in f\left(\sum_{i=1}^{n} a_{i}\right)+L_{0}=L_{0}$. The other direction might not hold. The same reasoning holds for tracts of [3].

(iv) Another interesting example comes from valuation theory. In [39, Definition 8.8(ii)], valuations are displayed as $\preceq$-morphisms of semirings, writing the target of the valuation as a semiring (using multiplicative notation instead of additive notation) via Green's relation of Example 2.16(viii). Here $\varphi\left(b_{1} b_{2}\right)=\varphi\left(b_{1}\right) \varphi\left(b_{2}\right)$. If we instead wrote $\varphi\left(b_{1} b_{2}\right) \preceq \varphi\left(b_{1}\right) \varphi\left(b_{2}\right)$, we would have a quasivaluation.

In conjunction with the hyperring theory, we are most interested in $\preceq$-morphisms and homomorphisms, but at times we need the restriction to homomorphisms. Occasional results can be formulated for $\succeq$ morphisms, such as in Lemma 3.11(iii) below.

2.3.1. Direct sums and direct limits.

The direct sum of $\mathcal{T}$-modules, defined in the usual way, is extended to pseudo-triples, 26, §2.5.1].

Definition 2.25. $\quad$ (i) The direct sum $\oplus_{i \in I}\left(\mathcal{A}_{i}, \mathcal{T}_{\mathcal{A}_{i}},(-)\right)$ of a family of pseudo-triples over an index set $I$ (not necessarily finite) is defined as $\left(\oplus \mathcal{A}_{i}, \mathcal{T}_{\oplus \mathcal{A}_{i}},(-)\right)$, where $\mathcal{T}_{\oplus \mathcal{A}_{i}}=\cup \mathcal{T}_{\mathcal{A}_{i}}$, viewed in $\oplus \mathcal{A}_{i}$.

(ii) The free $\mathcal{A}$-module pseudo-triple $\left(\mathcal{A}^{(I)}, \mathcal{T}_{\mathcal{A}^{(I)}},(-)\right)$ over a pseudo-triple $(\mathcal{A}, \mathcal{T},(-))$ is the direct sum of copies of $(\mathcal{A}, \mathcal{T},(-))$.

If $(\mathcal{A}, \mathcal{T},(-), \preceq))$ is a system, we can extend $\preceq$ componentwise to $\mathcal{A}^{(I)}$ to obtain the free $\mathcal{A}$-module system.

Remark 2.26. When $\preceq$ is a $P O$ on $\mathcal{A}$, $\preceq$ is also a $P O$ on $\mathcal{A}^{(I)}$, seen componentwise.

\section{Systemic Versions of BASIC MOdULE PROPERTIES}

We want to find the systemic generalization of classical concepts of module theory. As we shall see, this depends on which version we use, i.e., the switch negation map in the symmetrization given in $\$ 2.2$, or taking a given surpassing negation map $(-)$ and surpassing relation $\preceq$. These two different approaches give rise to different theories.

3.0.1. Notation.

Let us fix some notation for the remainder of this paper. In what follows, we let $\mathcal{A}=(\mathcal{A}, \mathcal{T},(-), \preceq)$ be a semiring system, and $\mathcal{M}$ and $\mathcal{N}$ always denote $\mathcal{A}$-systemic modules. We write $\preceq$ generically for the appropriate surpassing $\mathrm{PO}$ in a system.

\subsection{Module theoretic notions.}

Definition 3.1. Let $\mathcal{M}$ and $\mathcal{N}$ be $\mathcal{A}$-systemic modules, and $f: \mathcal{M} \rightarrow \mathcal{N}$ a $\preceq$-morphism.

(i) A submodule $\mathcal{M}^{\prime}$ of $\mathcal{M}$ is $f$-null if $f(a) \in \mathcal{N}_{\text {Null }}$ for all $a \in \mathcal{M}^{\prime}$. The null-module kernel $\operatorname{ker}_{\mathrm{Mod}, \mathcal{M}} f$ of $f$ is the sum of all $f$-null submodules of $\mathcal{M}$.

(ii) $A \preceq$-morphism $f: \mathcal{M} \rightarrow \mathcal{N}$ is null if $f(\mathcal{M}) \subseteq \mathcal{N}_{\text {Null }}$, i.e., $\operatorname{ker}_{\text {Mod.M }} f=\mathcal{M}$.

(iii) $A \preceq$-morphism $f$ is null-monic (resp. null-epic) when it satisfies the property that if $f h$ is null (resp. $h f$ is null) for a homomorphism $h$, then $h$ is null.

(iv) $A \preceq$-morphism $f$ is $N$-monic when it satisfies the property that if $f(b)=f\left(b^{\prime}\right)$ for $b, b^{\prime} \in \mathcal{M}$ then $b=b^{\prime}$.

Remark 3.2. $\quad$ (i) $\mathcal{M}_{\text {Null }} \subseteq \operatorname{ker}_{\text {Mod, } \mathcal{M}} f$, by Remark 2.22. 
(ii) Being the sum of submodules of $\mathcal{M}$, $\operatorname{ker}_{\mathrm{Mod}, \mathcal{M}} f$ is a submodule of $\mathcal{M}$, which is $f$-null when $f$ is a homomorphism, but need not be $f$-null when $f$ is just a $\preceq$-morphism. One could have $f\left(a_{1}\right)+f\left(a_{2}\right) \succeq \mathbb{0}$ whereas $f\left(a_{1}+a_{2}\right) \nsucceq \mathbb{0}$.

Lemma 3.3. $A \preceq$-morphism $f: \mathcal{M} \rightarrow \mathcal{N}$ is null-monic if and only if the null-module kernel of $f$ is a subset of $\mathcal{M}_{\text {Null }}$.

Proof. $(\Rightarrow)$ For any $f$-null submodule $\mathcal{M}^{\prime}$ of $\mathcal{M}$, consider the identity map $h: \mathcal{M}^{\prime} \rightarrow \mathcal{M}^{\prime}$. Then $f h$ is null, implying $h$ is null. In particular, $\mathcal{M}^{\prime}=\mathcal{M}_{\mathrm{Null}}^{\prime} \subseteq \mathcal{M}_{\text {Null }}$, and hence the null-module kernel of $f$ is a subset of $\mathcal{M}_{\text {Null }}$.

$(\Leftarrow)$ Suppose $f h$ is null, for a homomorphism $h: \mathcal{K} \rightarrow \mathcal{M}$. Then $f(h(\mathcal{K})) \subseteq \mathcal{N}_{\text {Null }}$. This implies that

$$
h(\mathcal{K}) \subseteq \operatorname{ker}_{\text {Mod, } \mathcal{M}} f \subseteq \mathcal{M}_{\text {Null }},
$$

proving that $f: \mathcal{M} \rightarrow \mathcal{N}$ is null-monic.

Next, we define some notation which we will use later in defining projective modules.

Definition 3.4. Let $f: \mathcal{M} \rightarrow \mathcal{N}$ be a $\preceq$-morphism of $\mathcal{A}$-systemic modules $\mathcal{M}$ and $\mathcal{N}$. We define the following two sets:

$f(\mathcal{M})_{\preceq}=\{b \in \mathcal{N}: b \preceq f(a)$ for some $a \in \mathcal{M}\}, \quad f(\mathcal{M})_{\succeq}=\{b \in \mathcal{N}: b \succeq f(a)$ for some $a \in \mathcal{M}\}$.

(i) $f: \mathcal{M} \rightarrow \mathcal{N}$ is $\preceq$-onto if $f(\mathcal{M})_{\preceq}=\mathcal{N}$, i.e., for every $b^{\prime} \in \mathcal{N}$ there exists $b \in \mathcal{M}$, for which $b^{\prime} \preceq f(b)$.

(ii) $f: \mathcal{M} \rightarrow \mathcal{N}$ is h-onto if $f$ is a $\preceq$-onto homomorphism.

(iii) $f: \mathcal{M} \rightarrow \mathcal{N}$ is $\succeq$-onto if $f(\mathcal{M})_{\succeq}=\mathcal{N}$, i.e., if for every $b^{\prime} \in \mathcal{N}$ there is $b \in \mathcal{M}$ such that $b^{\prime} \succeq f(b)$.

Although $\preceq$-onto and h-onto seem more appropriate for this paper, giving stronger results for projectivity, A. Connes and C. Consani $[8$ use a definition more in line with $\succeq$-onto and which seem more appropriate for homology, taking modules over the Boolean semiring $\mathbb{B}$, whose symmetrization has some properties parallel to the supertropical semialgebra. This connection is to be discussed in detail in [25].

Most of our results hold already for $\preceq$-onto $\preceq$-morphisms and homomorphisms.

Example 3.5. In the supertropical setting, $f: \mathcal{M} \rightarrow \mathcal{N}$ is $\preceq$-onto iff for every element $b$ of $\mathcal{N}$ there is $c \in \mathcal{M}$ such that $b+$ ghost $=f(c)$, which often is easy to satisfy when $c$ is a large enough ghost. $\succeq$-onto says that $b=f(c)+$ ghost, which for $b$ tangible says $b=f(c)$.

For fuzzy rings, the condition says something about how $f\left(K_{0}\right)$ sits inside $L_{0}$, notation as in [1] .

Lemma 3.6. Let $f: \mathcal{M} \rightarrow \mathcal{N}$ be a $\preceq$-morphism of $\mathcal{A}$-systemic modules $\mathcal{M}$ and $\mathcal{N}$. Then

(i) $f(\mathcal{M})_{\succeq}$ is a submodule of $\mathcal{N}$. Moreover, $f$ is $\succeq$-onto, if for every $b \in \mathcal{T}_{\mathcal{N}}$ there is a $\in \mathcal{M}$ such that $f(a) \preceq b$.

(ii) $f(\mathcal{M})_{\preceq}$ is a submodule of $\mathcal{N}$ for any homomorphism $f: \mathcal{M} \rightarrow \mathcal{N}$.

Proof. (i) $f(\mathcal{M})_{\succeq}$ is clearly closed under the action of $\mathcal{A}$ and contains $\mathbb{O}$. If $b_{i} \in f(\mathcal{M})_{\succeq}$ for $i=1,2$ then writing $b_{i} \succeq f\left(a_{i}\right)$, we have

$$
b_{1}+b_{2} \succeq f\left(a_{1}\right)+f\left(a_{2}\right) \succeq f\left(a_{1}+a_{2}\right) .
$$

This shows that $f(\mathcal{M})_{\succeq}$ is also closed under addition. The second assertion follows from the fact that $\mathcal{T}_{\mathcal{N}}$ generates $\mathcal{N}$. In fact, for any $b \in \mathcal{N}$, there exist $b_{i} \in \mathcal{T}_{\mathcal{N}}$ such that $b=\sum_{i} b_{i}$. But, from the given condition, we can find $a_{i} \in \mathcal{M}$ such that $f\left(a_{i}\right) \preceq b_{i}$ and hence we have

$$
b=\sum_{i} b_{i} \succeq \sum f\left(a_{i}\right) \succeq f\left(\sum_{i} a_{i}\right) .
$$

(ii) One can easily check that $f(\mathcal{M})_{\preceq}$ is closed under the action of $\mathcal{A}$ and contains $\mathbb{D}$. Suppose that $b_{1}, b_{2} \in f(\mathcal{M})_{\preceq}$, i.e., there exist $a_{1}, a_{2} \in \mathcal{M}$ such that $b_{i} \preceq f\left(a_{i}\right)$ for $i=1,2$. Since $f$ is a homomorphism, it follows that

$$
b_{1}+b_{2} \preceq f\left(a_{1}\right)+f\left(a_{2}\right)=f\left(a_{1}+a_{2}\right) .
$$

This shows that $b_{1}+b_{2} \in f(\mathcal{M})_{\preceq}$ and hence $f(\mathcal{M})_{\preceq}$ is also closed under addition. 
Definition 3.7. $\quad$ (i) An onto homomorphism $\pi: \mathcal{M} \rightarrow \mathcal{N}$ is an $N$-quasi-isomorphism if $\pi$ is also N-monic.

(ii) $A \preceq$-onto $\preceq$-morphism $\pi: \mathcal{M} \rightarrow \mathcal{N}$ is a $\preceq$-quasi-isomorphism if $\pi$ is also null-monic.

\subsection{Congruences.}

Recall that a congruence on $\mathcal{M}$ is an equivalence relation which preserves all of the operators; i.e., it is a subsystem of $\mathcal{M} \times \mathcal{M}$ that contains the diagonal $\operatorname{diag}_{\mathcal{M}}:=\{(a, a): a \in \mathcal{M}\}$ and is symmetric and transitive.

\section{3. $\preceq$-split and h-split epics.}

We recall a standard definition.

Definition 3.8. Let $\pi: \mathcal{M} \rightarrow \mathcal{N}$ be an onto homomorphism. We say that $\pi: \mathcal{M} \rightarrow \mathcal{N}$ splits if there is a homomorphism $\nu: \mathcal{N} \rightarrow \mathcal{M}$ such that $\pi \nu=\mathbb{1}_{\mathcal{N}}$.

In classical algebra, $\nu$ must be monic, and any split epic gives rise to an exact sequence.

Example 3.9. If $\mathcal{M}=\mathcal{N} \oplus \mathcal{N}^{\prime}$, then the canonical projection $\mathcal{M} \rightarrow \mathcal{N}$ splits via the natural injection $\nu: \mathcal{N} \rightarrow \mathcal{M}$.

This is trickier in the theory of systems since, as we shall see, the analog of splitting need not involve direct sums; a similar issue has been already observed in tropical algebra, cf. [36, §2]. Accordingly, we want to weaken the definition, and consider its implications.

We write $f \preceq g$ for $\preceq$-morphisms $f, g: \mathcal{M} \rightarrow \mathcal{N}$, if $f(b) \preceq g(b)$ for all $b \in \mathcal{M}$. Now, we weaken Definition 3.8 as follows:

\section{Definition 3.10.}

(i) We say that a $\preceq$-morphism $\pi: \mathcal{M} \rightarrow \mathcal{N} \preceq$-split $s$ (resp. h-splits) if there is a $\preceq$-morphism (resp. homomorphism) $\nu: \mathcal{N} \rightarrow \mathcal{M}$ such that $\mathbb{1}_{\mathcal{N}} \preceq \pi \nu$. In this case, we also say that $\nu$ $\preceq$-splits (resp. $\preceq h$-splits) $\pi$, and that $\mathcal{N}$ is a $\preceq$-retract (resp. h-retract) of $\mathcal{M}$. $\succeq$-splits (resp. $\succeq \boldsymbol{h}$-splits) is defined analogously, with $\mathbb{1}_{\mathcal{N}} \succeq \pi \nu$.

(ii) Let $f$ be any of $\{\preceq$-morphism, homomorphism $\succeq$-morphism, homomorphism $\}$.

(a) $f: \mathcal{M} \rightarrow \mathcal{M}$ is $\preceq$-idempotent if $f^{2} \succeq f$.

(b) $f: \mathcal{M} \rightarrow \mathcal{M}$ is $\mathcal{T}$-idempotent if $f^{2}(a)=f(a)$, for all $a \in \mathcal{T}$.

(c) $f: \mathcal{M} \rightarrow \mathcal{M}$ is $(\mathcal{T}, \preceq)$-idempotent if $f$ is both $\mathcal{T}$-idempotent and $\preceq$-idempotent.

Lemma 3.11. (i) If $\pi: \mathcal{M} \rightarrow \mathcal{N}$ and $\nu: \mathcal{N} \rightarrow \mathcal{M}$ are $\preceq$-morphisms with $\mathbb{1}_{\mathcal{N}} \preceq \pi \nu$, then $\pi$ is $\preceq$-onto, and $\nu \pi$ is $\preceq$-idempotent.

(ii) If $\pi: \mathcal{M} \rightarrow \mathcal{N}$ is a homomorphism and $\nu: \mathcal{N} \rightarrow \mathcal{M}$ is a $\preceq$-morphism with $\mathbb{1}_{\mathcal{N}} \preceq \pi \nu$, then $\mathbb{1}_{\mathcal{M}}(-) \nu \pi$ is $\preceq$-idempotent.

(iii) If $\pi: \mathcal{M} \rightarrow \mathcal{N}$ and $\nu: \mathcal{N} \rightarrow \mathcal{M}$ are $\succeq$-morphisms with $\mathbb{1}_{\mathcal{N}} \succeq \pi \nu$, then $\nu$ is null-monic and $\mathbb{1}(-) \nu \pi$ is $\preceq$-idempotent.

(iv) If $\pi: \mathcal{M} \rightarrow \mathcal{N}$ is a homomorphism and $\nu: \mathcal{N} \rightarrow \mathcal{M}$ is a $\preceq$-morphism with $a=\pi \nu(a)$ for all $a \in \mathcal{T}_{\mathcal{N}}$, then $\pi$ is onto, and $\nu \pi$ is $\mathcal{T}$-idempotent.

Proof. (i) For any $b \in \mathcal{N}$, we have that $b \preceq \pi(\nu(b))$. This shows that $\mathcal{N}=\pi(\mathcal{M})_{\preceq}$ and hence $\pi$ is $\preceq$-onto. Furthermore, $\nu \pi \nu \pi=\nu(\pi \nu) \pi \succeq \nu \mathbb{1}_{\mathcal{N}} \pi=\nu \pi$.

(ii) For any $b \in \mathcal{M}$, let $c=\pi(b)$, so $\left(\mathbb{1}_{\mathcal{M}}(-) \nu \pi\right)(b)=b(-) \nu(c)$. Also $\pi \nu(c) \succeq c$ implies $\pi \nu(c)(-) c \succeq \mathbb{0}$ by Lemma 2.11, so $c(-) \pi \nu(c) \succeq \mathbb{0}$, and thus $\nu(c(-) \pi \nu(c)) \succeq \mathbb{0}$. Hence

$$
\begin{aligned}
\left(\mathbb{1}_{\mathcal{M}}(-) \nu \pi\right)\left(\mathbb{1}_{\mathcal{M}}(-) \nu \pi\right)(b) & =\left(\mathbb{1}_{\mathcal{M}}(-) \nu \pi\right)(b(-) \nu(c)) \\
& =b(-) \nu(c)(-) \nu(c(-) \pi \nu(c)) \succeq b(-) \nu(c)=\left(\mathbb{1}_{\mathcal{M}}(-) \nu \pi\right)(b) .
\end{aligned}
$$

(iii) For the first assertion, from Lemma 3.3, it is enough to show that $\operatorname{ker}_{\text {Mod, } \mathcal{N}} \nu \subseteq \mathcal{N}_{\text {Null. }}$. If $b \in \operatorname{ker}_{\text {Mod, } \mathcal{N}} \nu$, then $\nu(b) \succeq \mathbb{0}$. Since $\mathbb{1}_{\mathcal{N}} \succeq \pi \nu$, we further have that

$$
b \succeq \pi \nu(b) \succeq \pi(\mathbb{0}) \succeq \mathbb{0}
$$

and hence $b \in \mathcal{N}_{\text {Null }}$, showing that $\operatorname{ker}_{\text {Mod, } \mathcal{N}} \nu \subseteq \mathcal{N}_{\text {Null. }}$. Also

$$
\left(\mathbb{1}_{\mathcal{M}}(-) \nu \pi\right)\left(\mathbb{1}_{\mathcal{M}}(-) \nu \pi\right) \succeq \mathbb{1}_{\mathcal{M}}+\nu \pi \nu \pi(-) \nu \pi(-) \nu \pi \succeq \mathbb{1}_{\mathcal{M}}+\nu \pi(-) \nu \pi(-) \nu \pi \succeq \mathbb{1}_{\mathcal{M}}(-) \nu \pi,
$$


showing that $\left(\mathbb{1}_{\mathcal{M}}(-) \nu \pi\right)$ is $\preceq$-idempotent.

(iv) For any $b \in \mathcal{N}$, we can write $b=\sum_{i} a_{i}$ for $a_{i} \in \mathcal{T}_{\mathcal{N}}$. Since, we assume that $a=\pi \nu(a)$ for any $a \in \mathcal{T}_{\mathcal{N}}$ and $\pi$ is a homomorphism, we have that

$$
b=\sum_{i} a_{i}=\sum_{i} \pi \nu\left(a_{i}\right)=\pi\left(\sum_{i} \nu\left(a_{i}\right)\right),
$$

showing that $\pi$ is onto. Furthermore, for any $b \in \mathcal{T}_{\mathcal{M}}$, we have that

$$
\nu \pi \nu \pi(b)=\nu(\pi \nu)(\pi(b))=\nu \pi(b),
$$

showing that $\nu \pi$ is $\mathcal{T}$-idempotent.

Definition 3.12. A systemic module $\mathcal{M}=\left(\mathcal{M}, \mathcal{T}_{\mathcal{M}},(-), \preceq\right)$ is the (finite) $\preceq$-direct sum of systemic modules $\left(\mathcal{M}_{i}, \mathcal{T}_{\mathcal{M}_{i}},(-), \preceq\right), \quad i \in I$ (I finite), if there are $\preceq$-morphisms $\pi_{i}: \mathcal{M} \rightarrow \mathcal{M}_{i}$ as well as $\preceq$ morphisms $\nu_{i}: \mathcal{M}_{i} \rightarrow \mathcal{M}$ that $\preceq$-split $\pi_{i}$, for which $\mathbb{1}_{\mathcal{M}} \preceq \sum \nu_{i} \pi_{i}$, $\mathbb{1}_{\mathcal{M}_{i}} \preceq \pi_{i} \nu_{i}$, and $\mathbb{O}_{\mathcal{M}} \preceq \pi_{j} \nu_{i}$ for all $i \neq j$.

The analogous definition, $\boldsymbol{h}$-direct sum, is for homomorphisms instead of $\preceq$-morphisms as follows:

$\left(\mathcal{M}, \mathcal{T}_{\mathcal{M}},(-), \preceq\right)$ is the (finite) $\boldsymbol{h}$-direct sum of $\left(\mathcal{M}_{i}, \mathcal{T}_{\mathcal{M}_{i}},(-), \preceq\right), \quad i \in I$ (I finite), if there are homomorphisms $\pi_{i}: \mathcal{M} \rightarrow \mathcal{M}_{i}$ as well as homomorphisms $\nu_{i}: \mathcal{M}_{i} \rightarrow \mathcal{M}$ that $\preceq$-split $\pi_{i}$, for which $\mathbb{1}_{\mathcal{M}} \preceq \sum \nu_{i} \pi_{i}, \mathbb{1}_{\mathcal{M}_{i}} \preceq \pi_{i} \nu_{i}$, and $\mathbb{0}_{\mathcal{M}} \preceq \pi_{j} \nu_{i}$ for all $i \neq j$.

$\mathcal{M}=\left(\mathcal{M}, \mathcal{T}_{\mathcal{M}},(-), \preceq\right)$ is the (finite) $\succeq$-direct sum of $\left(\mathcal{M}_{i}, \mathcal{T}_{\mathcal{M}_{i}},(-), \preceq\right), \quad i \in I$ (I finite), if there are $\succeq$-morphisms $\pi_{i}: \mathcal{M} \rightarrow \mathcal{M}_{i}$ as well as $\succeq$-morphisms $\nu_{i}: \mathcal{M}_{i} \rightarrow \mathcal{M}$ that $\succeq$-split $\pi_{i}$, for which $\mathbb{1}_{\mathcal{M}} \succeq \sum \nu_{i} \pi_{i}, \mathbb{1}_{\mathcal{M}_{i}} \succeq \pi_{i} \nu_{i}$, and $\mathbb{D}_{\mathcal{M}} \succeq \pi_{j} \nu_{i}$ for all $i \neq j$.

Then we have the following.

Theorem 3.13. Let $\pi: \mathcal{M} \rightarrow \mathcal{N}$ be a homomorphism. If $\nu \preceq$-splits $\pi$ (resp. h-splits $\pi$ ), then:

(i) $\mathcal{M}$ is the $\preceq$-direct sum (resp. h-direct sum) of $\mathcal{M}_{1}:=\pi(\mathcal{M})$ and $\mathcal{M}_{2}:=\left(\mathbb{1}_{\mathcal{M}}(-) \nu \pi\right)(\mathcal{M})$ with respect to the $\preceq$-morphisms (resp. homomorphisms) $\pi_{1}=\pi, \nu_{1}=\nu, \pi_{2}=\left(\mathbb{1}_{\mathcal{M}}(-) \nu \pi\right), \nu_{2}=\mathbb{1}_{\mathcal{M}_{2}}$.

(ii) $\mathcal{M}$ is the $\preceq$-direct sum (resp. h-direct sum) of $\mathcal{M}_{1}=\nu \pi(\mathcal{M})$ and $\mathcal{M}_{2}=\operatorname{ker}_{\operatorname{Mod}, \mathcal{M}} \pi$, with respect to $\nu_{i}=\mathbb{1}_{\mathcal{M}_{i}}$ for $i=1,2$.

Proof. For notational convenience, we write $\mathbb{1}_{\mathcal{M}}=\mathbb{1}_{\mathcal{M}_{i}}=\mathbb{1}$. $\mathbb{1}(-) \nu \pi$ is $\preceq$-idempotent, by Lemma 3.11(ii).

(i) $\pi_{1}$ is $\preceq$-onto by Lemma $3.11(\mathrm{i})$, and $\pi_{2}$ is $\preceq$-onto by the definition of $\mathcal{M}_{2}$.

Next, we show that $\nu_{2} \preceq$-splits $\pi_{2}$. Take $b_{2} \in \mathcal{M}_{2}$. This means that there exists $b_{1} \in \mathcal{M}$ such that $b_{2}=b_{1}(-) \nu \pi\left(b_{1}\right)$, and now one observes

$$
\pi_{2} \nu_{2}\left(b_{2}\right)=\pi_{2}\left(b_{2}\right)=(\mathbb{1}(-) \nu \pi)^{2}\left(b_{1}\right) \succeq(\mathbb{1}(-) \nu \pi)\left(b_{1}\right)=b_{2},
$$

since $\mathbb{1}(-) \nu \pi$ is $\preceq$-idempotent by Lemma 3.11 (ii).

We now show the remaining conditions. One can easily see the following:

$$
\nu_{1} \pi_{1}(b)+\nu_{2} \pi_{2}(b)=\nu \pi(b)+(\mathbb{1}(-) \nu \pi)(b)=b+(\nu \pi(b)(-) \nu \pi(b)) \succeq b, \quad b \in \mathcal{M},
$$

showing that $\mathbb{1}_{\mathcal{M}} \preceq \nu_{1} \pi_{1}+\nu_{2} \pi_{2}$.

Finally, we have for $b=(\mathbb{1}(-) \nu \pi) b^{\prime} \in \mathcal{M}_{2}$,

$$
\pi_{1} \nu_{2}(b)=\pi(\mathbb{1}(-) \nu \pi)\left(b^{\prime}\right)=(\pi(-) \pi \nu \pi)\left(b^{\prime}\right) \succeq \pi\left(b^{\prime}\right)(-) \pi\left(b^{\prime}\right) \succeq \mathbb{0},
$$

and similarly, for $b \in \mathcal{M}_{1}$,

$$
\pi_{2} \nu_{1}(b)=(\mathbb{1}(-) \nu \pi)(\nu(b))=\nu(b)(-) \nu \pi \nu(b) \succeq \nu(b)(-) \nu(b) \succeq 0 .
$$

The proof for h-splitting is analogous since $\pi_{i}$ and $\nu_{i}$ are homomorphisms.

(ii) The same sort of verifications as in (i), but easier. Now we take $\pi_{1}=\nu \pi, \pi_{2}=\mathbb{1}(-) \nu \pi$, and $\nu_{i}$ to be the canonical injection for $i=1,2$, which we write as the identity map. Then $\pi_{1}(\mathcal{M})=\mathcal{M}_{1}$ and, for every $b \in \mathcal{M}, \pi_{2}(b)=b(-) \nu \pi(b) \in \mathcal{M}_{2}$ since $\pi \nu \pi \succeq \pi$. But if $b_{2} \in \mathcal{M}_{2}$ then

$$
(\mathbb{1}(-) \nu \pi)\left(b_{2}\right)=b_{2}(-) \nu \pi\left(b_{2}\right) \succeq b_{2},
$$

so $\pi_{2}$ is $\preceq$-onto. 
$\nu_{2} \preceq$-splits $\pi_{2}$, since

$$
\pi_{2} \nu_{2}\left(b_{2}\right)=(\mathbb{1}(-) \nu \pi)\left(b_{2}\right)=b_{2}(-) \nu \pi\left(b_{2}\right) \succeq b_{2}(-) \mathbb{D} \succeq b_{2} .
$$

Furthermore, one can easily see that

$$
\nu_{1} \pi_{1}(b)+\nu_{2} \pi_{2}(b)=\nu \pi(b)+(\mathbb{1}(-) \nu \pi)(b)=b+(\nu \pi(b)(-) \nu \pi(b)) \succeq b, \quad b \in \mathcal{M} .
$$

Finally, for $b=(\mathbb{1}(-) \nu \pi) b^{\prime} \in \mathcal{M}_{2}$,

for $b=\nu \pi b^{\prime} \in \mathcal{M}_{1}$,

$$
\pi_{2} \nu_{1}(b)=(\mathbb{1}(-) \nu \pi)(b)=(\mathbb{1}(-) \nu \pi) \nu \pi\left(b^{\prime}\right) \succeq \nu \pi\left(b^{\prime}\right)(-) \nu \pi\left(b^{\prime}\right) \succeq \mathbb{O},
$$

and for $b \in \mathcal{M}_{2}, \pi_{1} \nu_{2}(b)=\pi(b) \succeq \mathbb{O}$.

\section{4. $\preceq$-PROJECTIVE AND $\succeq$-PROJECTIVE MODULES}

We are ready to define several versions of $\preceq$-projective systemic modules, as well as $\succeq$-projective modules over ground $\mathcal{T}$-systems. This encompasses results of [29], in view of Remark 2.14].

Definition 4.1. (See [10, 28, 29, 42] for comparison)

(i) A systemic module $\mathcal{P}:=\left(\mathcal{P}, \mathcal{T}_{\mathcal{P}},(-), \preceq\right)$ is projective if for any onto homomorphism of systemic modules $h: \mathcal{M} \rightarrow \mathcal{M}^{\prime}$, every homomorphism $f: \mathcal{P} \rightarrow \mathcal{M}^{\prime}$ lifts to a homomorphism $\tilde{f}: \mathcal{P} \rightarrow \mathcal{M}$, in the sense that $h \tilde{f}=f$.

(ii) $\mathcal{P}$ is $\preceq$-projective if for any $\preceq$-onto $\preceq$-morphism $h: \mathcal{M} \rightarrow \mathcal{M}^{\prime}$, every $\preceq$-morphism $f: \mathcal{P} \rightarrow \mathcal{M}^{\prime}$ $\preceq$-lifts to $a \preceq$-morphism $\tilde{f}: \mathcal{P} \rightarrow \mathcal{M}$, in the sense that $f \preceq h \tilde{f}$.

(iii) $\overline{\mathcal{P}}$ is $(\preceq, h)$-projective if for any $\preceq$-onto homomorphism $h: \mathcal{M} \rightarrow \mathcal{M}^{\prime}$, every $\preceq$-morphism $f: \mathcal{P} \rightarrow \mathcal{M}^{\prime} \preceq$-lifts to $a \preceq$-morphism $\tilde{f}: \mathcal{P} \rightarrow \mathcal{M}$, in the sense that $f \preceq h \tilde{f}$.

(iv) $\mathcal{P}$ is h-projective if for any $\preceq$-onto homomorphism $h: \mathcal{M} \rightarrow \mathcal{M}^{\prime}$, every homomorphism $f$ : $\mathcal{P} \rightarrow \mathcal{M}^{\prime} \preceq$-lifts to a homomorphism $\tilde{f}: \mathcal{P} \rightarrow \mathcal{M}$, in the sense that $f \preceq h \tilde{f}$.

(v) $\mathcal{P}$ is $\succeq$-projective is defined analogously to $\preceq$-projective, with $\succeq$ replacing $\preceq$ where appropriate. In other words, for any $\succeq$-onto $\preceq$-morphism $h: \mathcal{M} \rightarrow \mathcal{M}^{\prime}$, every $\succeq$-morphism $f: \mathcal{P} \rightarrow \mathcal{M}^{\prime}$ $\succeq$-lifts to $a \succeq$-morphism $\tilde{\tilde{f}}: \mathcal{P} \rightarrow \mathcal{M}$, in the sense that $f \succeq h \tilde{f}$.

Note that the subtleties in these versions: $\preceq$-projective implies $(\preceq, h)$-projective, but the condition could fail in the important case of free systems, cf. Remark 4.3 (ii) below. The definition for h-projective provides the most results, but these are less inclusive since $\preceq$-projective systems are more general, and still satisfy many results analogous to the projective theory.

\subsection{Basic properties of $\preceq$-projective and h-projective systems.}

Lemma 4.2. The free $\mathcal{A}$-systemic module $\mathcal{F}:=\mathcal{A}^{(I)}$ is projective, $(\preceq, h)$-projective, h-projective, and $\succeq$-projective.

Proof. We take the usual argument of lifting a set-theoretical map from the base $\left\{e_{i}: i \in I\right\}$ of $\mathcal{F}$, in these three respective contexts. Namely, choosing $x_{i} \in \mathcal{M}$ for which $h\left(x_{i}\right)=f\left(e_{i}\right)\left(\operatorname{resp} . h\left(x_{i}\right) \succeq f\left(e_{i}\right)\right.$, $\left.h\left(x_{i}\right) \preceq f\left(e_{i}\right)\right)$ and defining a homomorphism $\tilde{f}: \mathcal{F} \rightarrow \mathcal{M}$ by $\tilde{f}\left(e_{i}\right)=x_{i}$, we have the three respective comparisons:

$f\left(\sum a_{i} e_{i}\right)=\sum f\left(a_{i} e_{i}\right)=\sum a_{i} f\left(e_{i}\right)=\sum a_{i} h\left(x_{i}\right)=h\left(\sum a_{i} x_{i}\right)=h\left(\sum a_{i} \tilde{f}\left(e_{i}\right)\right)=h \tilde{f}\left(\sum a_{i} e_{i}\right)$ proving $f=h \tilde{f}$.

$f\left(\sum a_{i} e_{i}\right) \preceq \sum f\left(a_{i} e_{i}\right)=\sum a_{i} f\left(e_{i}\right) \preceq \sum a_{i} h\left(x_{i}\right)=h\left(\sum a_{i} x_{i}\right)=h\left(\sum a_{i} \tilde{f}\left(e_{i}\right)\right)=h \tilde{f}\left(\sum a_{i} e_{i}\right)$, proving $f \preceq h \tilde{f}$.

$f\left(\sum a_{i} e_{i}\right) \succeq \sum f\left(a_{i} e_{i}\right)=\sum a_{i} f\left(e_{i}\right) \succeq \sum a_{i} h\left(x_{i}\right) \succeq h\left(\sum a_{i} x_{i}\right)=h\left(\sum a_{i} \tilde{f}\left(e_{i}\right)\right)=h \tilde{f}\left(\sum a_{i} e_{i}\right)$ 
proving $f \succeq h \tilde{f}$.

For h-projective see Remark 4.3(i).

\section{Remark 4.3.}

(i) In the proof, we see, surprisingly, that any $\preceq$-morphism $f: \mathcal{F} \rightarrow \mathcal{M}$ can be $\preceq$-lifted to a homomorphism $\tilde{f}: \mathcal{F} \rightarrow \mathcal{M}$ (since $\tilde{f}$ is a homomorphism by definition). This proves the remaining case of h-projective in Lemma 4.2.

(ii) The free $\mathcal{A}$-module need not be $\preceq$-projective, since the satisfaction of the first two equations in the proof require $h$ to be a homomorphism!

4.1.1. Characterizations of $\preceq$-projective and h-projective systemic modules.

Similar arguments as in [17, §17] show that the following are equivalent for a systemic module $\mathcal{P}$ :

(i) $\mathcal{P}$ is projective.

(ii) Every homomorphism onto $\mathcal{P}$ splits.

(iii) There is an onto homomorphism from a free system to $\mathcal{P}$ that splits.

(iv) The functor $\operatorname{Hom}\left(\mathcal{P}, \_\right)$sends onto homomorphisms to onto homomorphisms.

(iii) is the condition used in [30] to define projective modules. We extend this to $\preceq$. Define $\operatorname{Mor}_{\preceq}(\mathcal{M}, \mathcal{N})$ to be the set of $\preceq$-morphisms from $\mathcal{M}$ to $\mathcal{N}$, and its subset $\operatorname{Hom}(\mathcal{M}, \mathcal{N})$ to be the homomorphisms.

Proposition 4.4. The following are equivalent for a systemic module $\mathcal{P}$ :

(i) $\mathcal{P}$ is $(\preceq, h)$-projective.

(ii) Every $\preceq$-onto homomorphism to $\mathcal{P} \preceq$-splits.

(iii) There is a $\preceq$-onto homomorphism from a free system to $\mathcal{P}$ that $\preceq$-splits.

(iv) Given a $\preceq$-onto $\preceq$-morphism $h: \mathcal{M} \rightarrow \mathcal{M}^{\prime}$, the map $\operatorname{Mor}_{\preceq}(\mathcal{P}, h): \operatorname{Mor}_{\preceq}(\mathcal{P}, \mathcal{M}) \rightarrow \operatorname{Mor}_{\preceq}\left(\mathcal{P}, \mathcal{M}^{\prime}\right)$ given by $g \mapsto h g$ is $\preceq$-onto.

Proof. $((i) \Rightarrow($ ii $))$ Given a $\preceq$-onto homomorphism $h: \mathcal{M} \rightarrow \mathcal{P}$, the identity map $1_{\mathcal{P}} \preceq$-lifts to a $\preceq$ morphism $g: \mathcal{P} \rightarrow \mathcal{M}$ satisfying $1_{\mathcal{P}} \preceq h g$.

$((i i) \Rightarrow($ iii $))$ A fortiori, since we can define a $\preceq$-onto homomorphism from a free system to $\mathcal{P}$ by taking a base $\left\{e_{i}\right\}$ of a free system and sending the $e_{i}$ elementwise to the $\preceq$-generators of $\mathcal{P}$ as in the proof of Lemma 4.2

$(($ iii $) \Rightarrow($ i $))$ Take a free systemic module $\mathcal{F}$, with the projection $\pi: \mathcal{F} \rightarrow \mathcal{P}$ which by hypothesis $\preceq$-splits, with $\nu: \mathcal{P} \rightarrow \mathcal{F}$. Let $h: \mathcal{M} \rightarrow \mathcal{M}^{\prime}$ be a $\preceq$-onto homomorphism. Then, for any $\preceq$-morphism $f: \mathcal{P} \rightarrow \mathcal{M}^{\prime}$, we can $\preceq$-lift $f \pi$ to $\tilde{f}: \mathcal{F} \rightarrow \mathcal{M}$, i.e., $f \pi \preceq h \tilde{f}$. Since $1_{\mathcal{P}} \preceq \pi \nu$, we have that

$$
f \preceq f(\pi \nu)=(f \pi) \nu \preceq h(\tilde{f} \nu),
$$

proving $\tilde{f} \nu \preceq$-lifts $f$.

$((i) \Leftrightarrow(i v))$ This directly follows from the definition. In fact, let $h: \mathcal{M} \rightarrow \mathcal{M}^{\prime}$ be a $\preceq$-onto homomorphism. Then we have:

$$
\operatorname{Mor}_{\preceq}(\mathcal{P}, h): \operatorname{Mor}_{\preceq}(\mathcal{P}, \mathcal{M}) \rightarrow \operatorname{Mor}_{\preceq}\left(\mathcal{P}, \mathcal{M}^{\prime}\right),
$$

given by $g \mapsto h g$. For notational convenience, let $\varphi:=\operatorname{Mor}_{\preceq}(\mathcal{P}, h), A:=\operatorname{Mor}_{\preceq}(\mathcal{P}, \mathcal{M})$, and $B:=$ $\operatorname{Mor}_{\preceq}\left(\mathcal{P}, \mathcal{M}^{\prime}\right)$. Then $\varphi$ is $\preceq$-onto if and only if $\varphi(A) \preceq=B$. Now, for any $f \in B$, since $\mathcal{P}$ is $(\preceq, h)$ projective, there exists $\tilde{f} \in A$, such that $f \preceq h \tilde{f}=\varphi(\tilde{f})$. This shows that $\varphi$ is $\preceq$-onto as desired.

Remark 4.5. One can easily see from the above proof on $((i) \Longrightarrow($ ii $))$ that if $\mathcal{P}$ is $\preceq$-projective then every $\preceq$-onto morphism to $\mathcal{P} \preceq$-splits.

More appropriate in later research is the h-version:

Proposition 4.6. The following are equivalent for a systemic module $\mathcal{P}$ :

(i) $\mathcal{P}$ is h-projective.

(ii) Every $\preceq$-onto homomorphism to $\mathcal{P}$ h-splits.

(iii) There is a $\preceq$-onto homomorphism from a free system to $\mathcal{P}$ that $h$-splits.

(iv) Given a $\preceq$-onto homomorphism $h: \mathcal{M} \rightarrow \mathcal{M}^{\prime}, \operatorname{Hom}(\mathcal{P}, h): \operatorname{Hom}(\mathcal{P}, \mathcal{M}) \rightarrow \operatorname{Hom}\left(\mathcal{P}, \mathcal{M}^{\prime}\right)$ given by $g \mapsto h g$ is $\preceq$-onto. 
Proof. The proof is analogous to that of Proposition 4.4. In fact, one may follow the proof of Proposition 4.4 by replacing $\preceq$-splits with $\mathrm{h}$-splits and also by using the fact that the composition $f g$ is a homomorphism if $f$ and $g$ are homomorphisms.

We also have the $\succeq$ version.

Proposition 4.7. The following are equivalent for a systemic module $\mathcal{P}$ :

(i) $\mathcal{P}$ is $\succeq$-projective.

(ii) Every $\succeq$-onto $\succeq$-morphism to $\mathcal{P} \succeq$-splits.

(iii) There is a $\succeq$-onto $\succeq$-morphism from a free system to $\mathcal{P}$ that $h$ - $\succeq$-splits.

(iv) The functor $\operatorname{Hom}\left(\mathcal{P}, \_\right)$sends $\succeq$-onto $\succeq$-morphisms to $\succeq$-onto $\succeq$-morphisms.

Proof. Analogous to the proof of Proposition 4.4 where we reverse $\preceq$ and $\succeq$ and apply Lemma 4.2 (taking $\succeq$-morphisms instead of homomorphisms).

Lemma 4.8 (as in [17, Proposition 17.19]). A direct sum $\sum \mathcal{P}_{i}$ of systemic modules is projective (resp. $\preceq$-projective, $\preceq$-h-projective, $\boldsymbol{h}$-projective, $\succeq$-projective) if and only if each $\mathcal{P}_{i}$ is projective (resp. $\preceq$-projective, $\preceq$-h-projective, $h$-projective, $\succeq$-projective).

Proof. Formal, according to components.

One can sharpen this assertion.

Proposition 4.9. If $\pi: \mathcal{Q} \rightarrow \mathcal{P}$ is a $\preceq$-split (resp. h-split) $\preceq$-morphism (resp. homomorphism) and $\mathcal{Q}$ is $\preceq$-projective (resp. h-projective), then $\mathcal{P}$ is also $\preceq$-projective (resp. h-projective).

Proof. We first prove the case when $\mathcal{Q}$ is $\preceq$-projective. We write a $\preceq$-splitting map $\nu: \mathcal{P} \rightarrow \mathcal{Q}$ as in Definition 3.10 . For any $\preceq$-onto morphism

$$
h: \mathcal{M} \rightarrow \mathcal{M}^{\prime}
$$

and every $\preceq$-morphism $f: \mathcal{P} \rightarrow \mathcal{M}^{\prime}$, the $\preceq$-morphism $f \pi \preceq$-lifts to a $\preceq$-morphism $\tilde{f}: \mathcal{Q} \rightarrow \mathcal{M}$, i.e., $h \tilde{f} \succeq f \pi$. Hence $h \tilde{f} \nu \succeq f \pi \nu \succeq f$, so $\tilde{f} \nu \preceq$-lifts $f$. This proves that $\mathcal{P}$ is $\preceq$-projective.

One may prove the h-split case by the analogous argument.

Proposition 4.10. Suppose $\mathcal{Q}$ is the $\preceq$-direct sum (resp. h-direct sum) of $\mathcal{P}_{i}$, with each $\mathcal{P}_{i}$ a $\preceq$-retract (resp. h-retract) of $\mathcal{Q}$. If the $\mathcal{P}_{i}$ are $(\preceq, h)$-projective (resp. h-projective) then $\mathcal{Q}$ is also $(\preceq, h)$-projective (resp. h-projective).

Proof. We write $\nu_{i}: \mathcal{P}_{i} \rightarrow \mathcal{Q}$ and $\pi_{i}: \mathcal{Q} \rightarrow \mathcal{P}_{i}$ as in Definition 3.12 For any $\preceq$-onto homomorphism $h: \mathcal{M} \rightarrow \mathcal{M}^{\prime}$ and $\preceq$-morphism $f: \mathcal{Q} \rightarrow \mathcal{M}^{\prime}$, define the $\preceq$-morphisms $f_{i}=f \nu_{i}: \mathcal{P}_{i} \rightarrow \mathcal{M}^{\prime}$, which $\preceq$-lift to $\preceq$-morphisms $\tilde{f}_{i}: \mathcal{P}_{i} \rightarrow \mathcal{M}$, i.e., $h \tilde{f}_{i} \succeq f \nu_{i}$. But then $h \tilde{f}_{i} \pi_{i} \succeq f \nu_{i} \pi_{i}$, so $h\left(\sum_{i} \tilde{f}_{i} \pi_{i}\right) \succeq f \sum_{i}\left(\nu_{i} \pi_{i}\right) \succeq f$, implying $\sum_{i} \tilde{f}_{i} \pi_{i} \preceq$-lifts $f$. The same argument holds for homomorphisms since a finite sum of homomorphisms is a homomorphism.

The corresponding result for $\succeq$-projective is proved analogously.

Proposition 4.11. Suppose $\mathcal{Q}$ is the $\succeq$-direct sum of $\mathcal{P}_{i}$, with each $\mathcal{P}_{i}$ a $\succeq$-retract of $\mathcal{Q}$. If the $\mathcal{P}_{i}$ are $\succeq$-projective then $\mathcal{Q}$ is also $\succeq$-projective.

Proof. We write $\nu_{i}: \mathcal{P}_{i} \rightarrow \mathcal{Q}$ and $\pi_{i}: \mathcal{Q} \rightarrow \mathcal{P}_{i}$ as in Definition 3.12 For any $\succeq$-onto homomorphism $h: \mathcal{M} \rightarrow \mathcal{M}^{\prime}$ and $\succeq$-morphism $f: \mathcal{Q} \rightarrow \mathcal{M}^{\prime}$, define the $\succeq$-morphisms $f_{i}=f \nu_{i}: \mathcal{P}_{i} \rightarrow \mathcal{M}^{\prime}$, which $\succeq$-lift to $\succeq$-morphisms $\tilde{f}_{i}: \mathcal{P}_{i} \rightarrow \mathcal{M}$, i.e., $h \tilde{f}_{i} \preceq f \nu_{i}$. But then $h \tilde{f}_{i} \pi_{i} \succeq f \nu_{i} \pi_{i}$, so $h\left(\sum_{i} \tilde{f}_{i} \pi_{i}\right) \preceq \sum_{i} h \tilde{f}_{i} \pi_{i} \preceq$ $f \sum_{i}\left(\nu_{i} \pi_{i}\right) \preceq f$, implying $\sum_{i} \tilde{f}_{i} \pi_{i} \succeq$-lifts $f$.

Proposition 4.12. If $\mathcal{Q}$ is $\preceq$-quasi-isomorphic to $\mathcal{P}_{1}$ and $\mathcal{P}_{1}$ is $(\preceq, h)$-projective, then $\mathcal{Q}$ is also $(\preceq, h)$ projective.

Proof. The same proof as before. We take the $\preceq$-quasi-isomorphism $\pi: \mathcal{Q} \rightarrow \mathcal{P}_{1}$. By $(\preceq, h)$-projectivity, there is a $\preceq$-retract $\nu: \mathcal{P}_{1} \rightarrow \mathcal{Q}$. For any $\preceq$-onto homomorphism $h: \mathcal{M} \rightarrow \mathcal{M}^{\prime}$ any $\preceq$-morphism $f: \mathcal{Q} \rightarrow \mathcal{M}^{\prime} \preceq$-lifts to a homomorphism $\tilde{f}: \mathcal{P}_{1} \rightarrow \mathcal{M}$, i.e., $h \tilde{f} \succeq f \nu$. But then $h \tilde{f} \pi \succeq f \nu \pi$, so in view of Lemma 3.3, $h(\tilde{f} \pi) \succeq f$, implying $\tilde{f} \pi \preceq$-lifts $f$. 
In [20] a stronger version of projectivity is used in the tropical theory, studied intensively in [30], namely,

Definition 4.13. A systemic module is strongly projective if it is a direct summand of a free systemic module.

An example was given in 20 of a projective module that is not strongly projective.

Remark 4.14 (cf. [17, Proposition 17.14]). Every strongly projective systemic module is projective, $\preceq$ projective, $h$-projective, and $\succeq$-projective, seen by passing to the free systemic module and appealing to Lemma 4.2 and Lemma 4.8. The theory of strongly projective modules is nice, but too restrictive for our purposes for homology.

Theorem 4.15. If $\mathcal{P}_{1}$ is $(\preceq, h)$-projective with a $\preceq$-onto homomorphism $\pi: \mathcal{P} \longrightarrow \mathcal{P}_{1}$ whose null-module kernel $\mathcal{K}$ is $(\preceq, h)$-projective, then $\mathcal{P}$ also is $(\preceq, h)$-projective.

Proof. We first lift the identity map of $\mathcal{P}_{1}$ to a $\preceq$-retract $\nu: \mathcal{P}_{1} \rightarrow \mathcal{P}$ of $\pi$, and let $\pi_{2}=\mathbb{1}_{\mathcal{P}}(-) \nu \pi$, which is $\preceq$-idempotent by Lemma 3.11(ii). Consider a $\preceq$-onto homomorphism $h: \mathcal{M} \rightarrow \mathcal{M}^{\prime}$. Then for any $\preceq$-morphism $f: \mathcal{P} \rightarrow \mathcal{M}^{\prime}$, we lift $f \nu: \mathcal{P}_{1} \rightarrow \mathcal{M}^{\prime}$ past $h$ to a $\preceq$-morphism $\tilde{f}_{1}: \mathcal{P}_{1} \rightarrow \mathcal{M}$. Next, we lift $\left.f\right|_{\mathcal{K}}: \mathcal{K} \rightarrow \mathcal{M}^{\prime}$ to a $\preceq$-morphism $\tilde{f}_{2}: \mathcal{K} \rightarrow \mathcal{M}$. We claim that for any $b \in \mathcal{P}, \pi_{2}(b) \in \mathcal{K}$. In fact, for any $b \in \mathcal{P}, \pi(b)(-) \pi \nu \pi(b) \succeq \pi(b)(-) \pi(b) \succeq \mathbb{0}$, so

$$
\pi\left(\pi_{2}(b)\right)=\pi\left(\mathbb{1}_{\mathcal{P}}(-) \nu \pi\right)(b)=\pi(b(-) \nu \pi(b))=\pi(b)(-) \pi \nu \pi(b) \succeq \pi(b)(-) \pi(b) \succeq \mathbb{0} .
$$

This implies that $\pi\left(\pi_{2}(b)\right) \in\left(\mathcal{P}_{1}\right)$ Null and hence $\pi_{2}(b) \in \mathcal{K}$. Now, we define a $\preceq$-morphism $\tilde{f}: \mathcal{P} \rightarrow \mathcal{M}$ as follows:

$$
\tilde{f}(b)=\tilde{f}_{1}(\pi(b))+\tilde{f}_{2}\left(\pi_{2}(b)\right) .
$$

Then $\tilde{f}$ is well-defined since $\pi_{2}(b) \in \mathcal{K}$. For any $b$ in $\mathcal{P}$ we have, using Definition 2.21 (iii,v),

$$
\begin{aligned}
h \tilde{f}(b) & =h \tilde{f}_{1}(\pi(b))+h \tilde{f}_{2}(\mathbb{1}(-) \nu \pi)(b) \\
& \succeq f(\nu \pi(b))+f((\mathbb{1}(-) \nu \pi)(b)) \succeq f(b+\nu \pi(b)(-) \nu \pi(b)) \succeq f(b),
\end{aligned}
$$

proving $h \tilde{f} \succeq f$, i.e. $\tilde{f} \preceq$-lifts $f$.

\subsection{2. $\preceq$-idempotent and $\preceq$-von Neumann regular matrices.}

Recall that an $m \times n$ matrix $A$ (with entries in a commutative ring) is said to be von Neumann regular if there exists a matrix $B$ such that $A=A B A$. Classically, there is a well-known correspondence among von Neumann regularity, idempotency, and projectivity. In the tropical setting, as pointed out in [19], projectivity can be expressed in terms of idempotent and von Neumann regular matrices.

In what follows, we assume that all matrices have entries in a system $\mathcal{A}$ unless otherwise stated. We generalize the aforementioned correspondence to the $\preceq$-version.

Definition 4.16. We say $A \preceq B$ for $m \times n$ matrices $A=\left(a_{i, j}\right), B=\left(b_{i, j}\right)$, if $a_{i, j} \preceq b_{i, j}$ for all $i, j$.

An $n \times n$ matrix $A$ is $\preceq$-idempotent if $A \preceq A^{2}$.

An $m \times n$ matrix $A$ is $\preceq$-von Neumann regular if there is an $n \times m$ matrix $B$ for which $A \preceq A B A$.

Proposition 4.17. Suppose $A$ is $\preceq$-idempotent. Then the module $A \mathcal{F}$ is $\preceq$-projective; in other words the column space of $A$ is a $\preceq$-projective $\mathcal{A}$-submodule of $\mathcal{F}$, and symmetrically the row space of $A$ is a $\preceq$-projective $\mathcal{A}$-submodule of $\mathcal{F}$.

Proof. Define $\pi: \mathcal{F} \rightarrow A \mathcal{F}$ by $\pi(v)=A v$. Then $\pi \preceq \pi^{2}$, and taking $\nu: A \mathcal{F} \rightarrow \mathcal{F}$ to be the identity, we have $\mathbb{1} \preceq \pi \nu$ on $A \mathcal{F}$, so we conclude by Proposition 4.9.

Corollary 4.18. If $A \preceq A B A$, then $A B \mathcal{F}$ is $\preceq$-projective.

Proof. $A B$ is $\preceq$-idempotent, since $A B \preceq(A B)^{2}=(A B A) B$.

Example 4.19. Proposition 4.17 gives us an explicit way of obtaining new $\preceq$-projectives, via $\preceq$-idempotent matrices. For example, if (-) is of the first kind (which happens in the supertropical case, see [39, Definition 2.22] for the definition), and $A^{\prime}$ is an idempotent matrix, then

$$
\left(I+A^{\prime}\right)^{2}=I+\left(A^{\prime}\right)^{\circ}+\left(A^{\prime}\right)^{2} \succeq I+A^{\prime}
$$


and this can be done in general.

The analogous results hold for $\succeq$-idempotent and $\succeq$-von Neumann regular. This raises the question of whether $A B \mathcal{F}=A \mathcal{F}$ when $A \preceq A B A$. Clearly $A \mathcal{F} \preceq A B A \mathcal{F} \subseteq A B \mathcal{F} \subseteq A \mathcal{F}$, which often implies equality, but a thorough discussion would take us too far afield here.

Trlifaj [43] has considered the dual to Baer's criterion:

We say a systemic module $\mathcal{M}$ is finitely $\preceq$-generated (as a systemic module ) if it is $\preceq$-generated by a finite set of cyclic systemic modules.

Remark 4.20 (As in 43, p. 2]). Suppose for any $\preceq$-onto homomorphism $h: \mathcal{M} \rightarrow \mathcal{M}^{\prime}$ of systemic modules, with $\mathcal{M}$ cyclic, that every $\preceq$-morphism $f: \mathcal{P} \rightarrow \mathcal{M}^{\prime} \preceq$-lifts to a $\preceq$-morphism $\tilde{f}: \mathcal{P} \rightarrow \mathcal{M}$. Then this condition holds for $\mathcal{M} \preceq$-finitely generated. (Indeed, write $\mathcal{M} \preceq \sum_{i=1}^{\bar{t}} \mathcal{A} a_{i}$ for $a_{i} \in \mathcal{M}$, apply the criterion for each $\mathcal{A} a_{i}$, and add the $\preceq$-liftings, i.e., $\left.\tilde{f}(a)=\sum \tilde{f}_{i}(a)\right)$.

[43. Lemma 2.1] gives a countable counterexample to this condition, and presents a readable and interesting account of the dual Baer criterion in the classical case.

\subsection{The $\prec$-dual basis lemma.}

Deore and Pati 10 proved a dual basis lemma for projective modules, and the same proof works for $\preceq$-projectives, h-projectives, and $\succeq$-projectives.

Proposition 4.21. A module pseudo-system $\left(\mathcal{P}, \mathcal{T}_{\mathcal{P}},(-), \preceq\right)$ that is $\preceq$-generated by $\left\{p_{i} \in \mathcal{P}: i \in I\right\}$ is $(\preceq, h)$-projective (resp. h-projective) if and only if there are $\preceq$-onto $\preceq$-morphisms (resp. homomorphisms) $g_{i}: \mathcal{P} \rightarrow \mathcal{A}$ such that for all $a \in \mathcal{A}$ we have $a \preceq \sum g_{i}(a) p_{i}$, where $g_{i}(a)=\mathbb{O}$ for all but finitely many $i$.

Proof. The assertion can be copied almost word for word from the standard proof, for example from [38, p. 493]. We take the free systemic module $\mathcal{F}=\left(\mathcal{A}^{(I)}, \mathcal{T}^{(I)},(-), \preceq\right)$ with base $\left\{e_{i}: i \in I\right\}$, and the $\preceq$-onto homomorphism $f: \mathcal{F} \rightarrow \mathcal{P}$ given by $f\left(e_{i}\right)=p_{i}, \forall i \in I$. Also we define the canonical projections $\pi_{j}: \mathcal{F} \rightarrow \mathcal{A}$ by $\pi_{j}\left(e_{i}\right)=\delta_{i j}$. Thus $c=\sum \pi_{i}(c) e_{i}$ for any $c \in \mathcal{F}$.

$(\Rightarrow)$ In view of Proposition 4.4 (or 4.6), $f$ is $\preceq$-split (resp. h-split), so we take a $\preceq$-morphism (resp. homomorphism) $g: \mathcal{P} \rightarrow \mathcal{F}$ with $f g \succeq 1_{\mathcal{P}}$. Put $g_{i}=\pi_{i} g: \mathcal{P} \rightarrow \mathcal{A}$. Then any $a \in \mathcal{P}$ satisfies

$$
\begin{aligned}
a & \preceq f g(a)=f\left(\sum_{i} \pi_{i}(g(a)) e_{i}\right) \\
& =\sum_{i} f\left(g_{i}(a) e_{i}\right)=\sum_{i} g_{i}(a) f\left(e_{i}\right)=\sum_{i} g_{i}(a) p_{i},
\end{aligned}
$$

as desired. If $g$ is a homomorphism then each $g_{i}$ is a homomorphism, seen by checking components.

$(\Leftarrow)$ Defining $g: \mathcal{P} \rightarrow \mathcal{F}$ by $g(a)=\sum g_{i}(a) e_{i}$, we have

$$
f g(a)=\sum g_{i}(a) f\left(e_{i}\right)=\sum g_{i}(a) p_{i} \succeq a .
$$

Thus $f g \succeq 1_{\mathcal{P}}$, so $\mathcal{P}$ is $(\preceq, h)$-projective, by Proposition 4.4 . When each of the $g_{i}$ is a homomorphism then clearly $g$ is a homomorphism.

Proposition 4.22. Suppose a module pseudo-system $\left(\mathcal{P}, \mathcal{T}_{\mathcal{P}},(-), \preceq\right)$ is generated by $\left\{p_{i} \in \mathcal{P}: i \in I\right\}$. Then $\mathcal{P}$ is $(\succeq, h)$-projective if and only if there are $\succeq$-onto $\succeq$-morphisms $g_{i}: \mathcal{P} \rightarrow \mathcal{A}$ such that for all $a \in \mathcal{A}$ we have $a \succeq \sum g_{i}(a) p_{i}$, where $g_{i}(a)=\mathbb{O}$ for all but finitely many $i$.

Proof. The analogous argument to the proof of Proposition 4.21 works, where $\preceq$ and $\succeq$ are interchanged.

\section{Versions of Schanuel's Lemma}

We turn to systemic versions of Schanuel's Lemma which could eventually relate to systemic projective dimension (which is work in progress). In the classical case, one reduces to the following case: Given two

exact sequences $\mathcal{K} \rightarrow \mathcal{P} \stackrel{f}{\rightarrow} \mathcal{M}$ and $\mathcal{K}^{\prime} \rightarrow \mathcal{P}^{\prime} \stackrel{f^{\prime}}{\rightarrow} \mathcal{M}$, with $f, f^{\prime}$ epic and $\mathcal{P}, \mathcal{P}^{\prime}$ projective, one concludes that $\mathcal{P} \oplus \mathcal{K}^{\prime}=\mathcal{P}^{\prime} \oplus \mathcal{K}$. However, for modules over general semirings, one cannot expect this to hold. 
The right notion of exactness for modules over semirings is rather subtle. Still, one can mimic the standard proof [33, pp. 165-167] of Schanuel's Lemma for modules over rings, by considering our more general version of splitting, and avoiding mixing submodules with kernels of homomorphisms (which are congruences). To this end, we introduce the following definition of congruence kernels.

Definition 5.1. Let $f: \mathcal{M} \rightarrow \mathcal{N}$ be $a \preceq$-morphism.

(i) The $\boldsymbol{N}$-congruence kernel $\operatorname{ker}_{N} f$ of $f$ is defined to be the following set:

$$
\operatorname{ker}_{N} f:=\left\{\left(a_{0}, a_{1}\right) \in \mathcal{M} \times \mathcal{M}: f\left(a_{0}\right)=f\left(a_{1}\right)\right\} .
$$

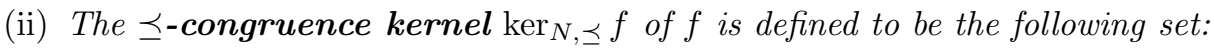

$$
\operatorname{ker}_{N, \preceq} f:=\left\{\left(a_{0}, a_{1}\right) \in \mathcal{M} \times \mathcal{M}: f\left(a_{0}\right)=f\left(a_{1}\right), \quad f\left(a_{0}\right), f\left(a_{1}\right) \in \mathcal{N}_{N u l l}\right\} .
$$

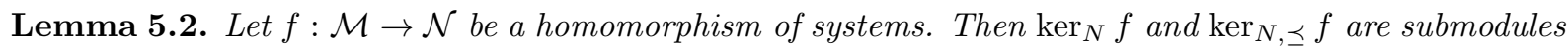
of $\mathcal{M} \times \mathcal{M}$. Also, $\operatorname{ker}_{N} f$ and $\operatorname{ker}_{N, \preceq f}$ are congruences on $\mathcal{M}$.

Proof. This is clear.

Theorem 5.3 (Semi-Schanuel). Suppose we have $\preceq$-morphisms $\mathcal{P}_{1} \stackrel{f_{1}}{\longrightarrow} \mathcal{M}$ and $\mathcal{P}_{2} \stackrel{f_{2}}{\longrightarrow} \mathcal{M}$ with $f_{1}$ and $f_{2}$ onto. (We are not assuming that either $\mathcal{P}_{i}$ is projective.) Let

$$
\mathcal{P}=\left\{\left(b_{1}, b_{2}\right): \quad b_{i} \in \mathcal{P}_{i}, f_{1}\left(b_{1}\right)=f_{2}\left(b_{2}\right)\right\},
$$

a submodule of $\mathcal{P}_{1} \oplus \mathcal{P}_{2}$, together the restriction $\pi_{i}^{\text {res }}$ of the projection $\pi_{i}: \mathcal{P} \rightarrow \mathcal{P}_{i}$ on the $i$ coordinate, for $i=1,2$.

(i) $\pi_{1}^{\text {res }}: \mathcal{P} \rightarrow \mathcal{P}_{1}$ is an onto homomorphism and and there is an onto homomorphism

$$
\operatorname{ker}_{N} \pi_{1}^{\mathrm{res}} \rightarrow \operatorname{ker}_{N} f_{2},
$$

(This part is purely semiring-theoretic and does not require a system.)

(ii) The maps $f_{1} \pi_{1}^{\text {res }}, f_{2} \pi_{2}^{\text {res }}: \mathcal{P} \rightarrow \mathcal{M}$ are the same.

(iii) In the systemic setting, $\pi_{1}^{\text {res }}$ also induces $\preceq$-quasi-isomorphism

$$
\pi_{N, \preceq}: \operatorname{ker}_{N, \preceq} \pi_{1}^{\text {res }} \rightarrow \operatorname{ker}_{N, \preceq} f_{2} .
$$

(iv) In (iii), if $f_{1}$ also is null-monic, we have the following $\preceq$-quasi-isomorphism:

$$
\operatorname{ker}_{N, \preceq} f_{1} \pi_{1}^{\text {res }} \rightarrow \operatorname{ker}_{N, \preceq f_{2}} .
$$

(v) If $\mathcal{P}_{1}$ is projective, then it is a retract of $\mathcal{P}$ with respect to the projection $\pi_{1}: \mathcal{P} \rightarrow \mathcal{P}_{1}$.

(vi) If $\mathcal{P}_{1}$ is $\preceq$-projective, then it is a $\preceq$-retract of $\mathcal{P}$ with respect to the projection $\pi_{1}: \mathcal{P} \rightarrow \mathcal{P}_{1}$, and $\mathcal{P}$ is the $\preceq$-direct sum of $\mathcal{P}_{1}$ and $\left(\mathbb{1}_{\mathcal{P}}(-) \nu_{1} \pi_{1}\right)(\mathcal{P})$.

Proof. Clearly $\mathcal{P}$ is a submodule of $\mathcal{P}_{1} \oplus \mathcal{P}_{2}$. We modify the standard proof.

(i) We first prove that $\pi_{1}^{\text {res }}$ is an onto homomorphism. In fact, clearly $\pi_{1}$ is a homomorphism since it is a projection and hence its restriction $\pi_{1}^{\text {res }}$ is also a homomorphism. Now, since $f_{2}$ is onto, for any $b_{1} \in \mathcal{P}_{1}$ there is $b_{2} \in \mathcal{P}_{2}$ such that $f_{1}\left(b_{1}\right)=f_{2}\left(b_{2}\right)$, implying $\left(b_{1}, b_{2}\right) \in \mathcal{P}$. Hence $\pi_{1}$ restricts to an onto homomorphism $\pi_{1}^{\text {res }}: \mathcal{P} \rightarrow \mathcal{P}_{1}$. For the remaining part of (i), one can easily see that

$$
\operatorname{ker}_{N} \pi_{1}^{\mathrm{res}} \subseteq\left\{\left(\left(b_{1}, b_{2}\right),\left(b_{1}^{\prime}, b_{2}^{\prime}\right)\right): b_{1}=b_{1}^{\prime}\right\}
$$

But, by the definition of $\pi_{1}^{\text {res }}$ and $\mathcal{P}$, we have that, for $\left(\left(b_{1}, b_{2}\right),\left(b_{1}, b_{2}^{\prime}\right)\right) \in \operatorname{ker}_{N} \pi_{1}^{\text {res }}$,

$$
f_{2}\left(b_{2}\right)=f_{1}\left(b_{1}\right)=f_{2}\left(b_{2}^{\prime}\right)
$$

which means that $\left(b_{2}, b_{2}^{\prime}\right) \in \operatorname{ker}_{N} f_{2}$. In other words,

$$
\operatorname{ker}_{N} \pi_{1}^{r e s}=\left\{\left(\left(b_{1}, b_{2}\right),\left(b_{1}, b_{2}^{\prime}\right)\right): b_{1} \in \mathcal{P}_{1},\left(b_{2}, b_{2}^{\prime}\right) \in \operatorname{ker}_{N} f_{2}\right\} .
$$

We define an onto homomorphism as follows:

$$
\pi: \operatorname{ker}_{N} \pi_{1}^{r e s} \rightarrow \operatorname{ker}_{N} f_{2}, \quad\left(\left(b_{1}, b_{2}\right),\left(b_{1}, b_{2}^{\prime}\right)\right) \mapsto\left(b_{2}, b_{2}^{\prime}\right),
$$

where $b_{1} \in \mathcal{P}_{1}$.

(ii) $f_{1} \pi_{1}^{\text {res }}\left(b_{1}, b_{2}\right)=f_{1}\left(b_{1}\right)=f_{2}\left(b_{2}\right)=f_{2} \pi_{2}^{r e s}\left(b_{1}, b_{2}\right)$. 


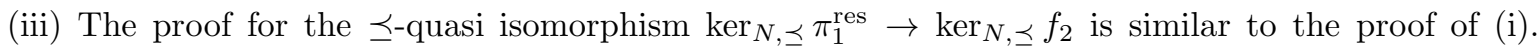
Notice that $\operatorname{ker}_{N, \preceq} \pi_{1}^{\text {res }}$ consists of those pairs $\left(\left(b_{1}, b_{2}\right),\left(b_{1}^{\prime}, b_{2}^{\prime}\right)\right)$ in $\mathcal{P} \times \mathcal{P}$ for which $b_{1}=b_{1}^{\prime} \in \mathcal{P}_{1, \text { Null }}$. But then

$$
f_{2}\left(b_{2}\right)=f_{1}\left(b_{1}\right)=f_{1}\left(b_{1}^{\prime}\right)=f_{2}\left(b_{2}^{\prime}\right),
$$

which are all in $\mathcal{M}_{\text {Null }}$ since $f_{1}\left(b_{1}\right) \in \mathcal{M}_{\text {Null }}$, implying $\left(b_{2}, b_{2}^{\prime}\right) \in \operatorname{ker}_{N, \preceq} f_{2}$. We define the $\preceq$-morphism $\pi_{N, \preceq}: \operatorname{ker}_{N, \preceq} \pi_{1}^{\text {res }} \rightarrow \operatorname{ker}_{N, \preceq} f_{2}$ by

$$
\left(\left(b_{1}, b_{2}\right),\left(b_{1}^{\prime}, b_{2}^{\prime}\right)\right) \mapsto\left(b_{2}, b_{2}^{\prime}\right) .
$$

Suppose that $\left(b_{2}, b_{2}^{\prime}\right) \in \operatorname{ker}_{N, \preceq} f_{2}$, i.e., $f_{2}\left(b_{2}\right)=f_{2}\left(b_{2}^{\prime}\right) \in \mathcal{M}_{\text {Null }}$. Since $f_{1}$ is onto, we can find $b_{1}, b_{1}^{\prime}$ in $\mathcal{P}_{1}$ such that $f_{1}\left(b_{1}\right)=f_{2}\left(b_{2}\right)$ and $f_{1}\left(b_{1}^{\prime}\right)=f_{2}\left(b_{2}^{\prime}\right)$. Clearly, for any $\left(b_{2}, b_{2}^{\prime}\right) \in \operatorname{ker}_{N, \preceq} f_{2}$, we have that

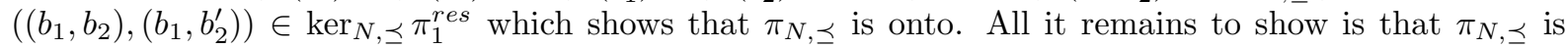
null-monic. Suppose that

$$
\pi_{N, \preceq}\left(\left(b_{1}, b_{2}\right),\left(b_{1}, b_{2}^{\prime}\right)\right)=\left(b_{2}, b_{2}^{\prime}\right) \in\left(\operatorname{ker}_{N, \preceq} f_{2}\right)_{\mathrm{Null}} .
$$

This means that $b_{2}, b_{2}^{\prime} \in\left(\mathcal{P}_{2}\right)_{\text {Null }}$. It follows that $\left(\left(b_{1}, b_{2}\right),\left(b_{1}, b_{2}^{\prime}\right)\right)$ is an element of $\left(\operatorname{ker}_{N, \preceq} \pi_{1}^{r e s}\right)_{\text {Null }}$,

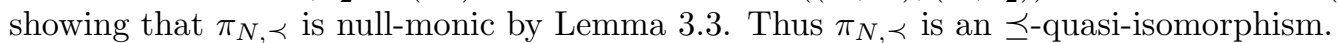

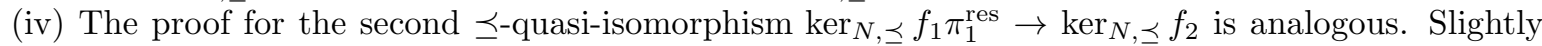
abusing notation, we define the following $\preceq$-morphism:

$$
\pi_{\preceq}: \operatorname{ker}_{N, \preceq} f_{1} \pi_{1}^{\mathrm{res}} \rightarrow \operatorname{ker}_{N, \preceq} f_{2}, \quad\left(\left(b_{1}, b_{2}\right),\left(b_{1}^{\prime}, b_{2}^{\prime}\right)\right) \mapsto\left(b_{2}, b_{2}^{\prime}\right) .
$$

One can easily see that $\pi \preceq$ is null-monic from exactly the same argument as above, along with the hypothesis that $f_{1}$ is null-monic. Now, suppose that $\left(b_{2}, b_{2}^{\prime}\right) \in \operatorname{ker}_{N, \preceq} f_{2}$. In other words, we have that $f_{2}\left(b_{2}\right)=f_{2}\left(b_{2}^{\prime}\right)$ and $f_{2}\left(b_{2}\right), f_{2}\left(b_{2}^{\prime}\right) \in \mathcal{M}_{\text {Null }}$. Again, since $f_{1}$ is onto, we can find an element $\alpha:=$ $\left(\left(b_{1}, b_{2}\right),\left(b_{1}^{\prime}, b_{2}^{\prime}\right)\right) \in \mathcal{P} \times \mathcal{P}$. We claim that $\alpha \in \operatorname{ker}_{N, \preceq} f_{1} \pi_{1}^{\text {res}} ;$ in this case, $\pi_{\preceq}(\alpha)=\left(b_{2}, b_{2}^{\prime}\right)$, showing that $\pi_{\preceq}$ is onto. In fact, we have

$$
f_{1} \pi_{1}^{\mathrm{res}}\left(b_{1}, b_{2}\right)=f_{1}\left(b_{1}\right)=f_{2}\left(b_{2}\right)=f_{2}\left(b_{2}^{\prime}\right)=f_{1}\left(b_{1}^{\prime}\right)=f_{1} \pi_{1}^{\mathrm{res}}\left(b_{1}^{\prime}, b_{2}^{\prime}\right) .
$$

Furthermore, since $f_{2}\left(b_{2}\right) \in \mathcal{M}_{\text {Null }}$, we have that $f_{1} \pi_{1}^{\text {res }}\left(b_{1}, b_{2}\right), f_{1} \pi_{1}^{\text {res }}\left(b_{1}^{\prime}, b_{2}^{\prime}\right) \in \mathcal{M}_{\text {Null }}$, proving our claim.

(v) Since $\mathcal{P}_{1}$ is projective and $\pi_{1}: \mathcal{P} \rightarrow \mathcal{P}_{1}$ is onto, $\pi_{1}$ splits via $\nu_{1}$ with $\pi_{1} \nu_{1}=1$.

(vi) Take $\nu_{1}: \mathcal{P}_{1} \rightarrow \mathcal{P}$ to be a $\preceq$-morphism $\preceq$-splitting $\pi_{1}$ via the identity map on $\mathcal{P}_{1}$, and we can apply Theorem 3.13 since $\pi_{1}$ is a homomorphism. delete.

The proof of (iv) seems to require a rather strong hypothesis, which it would be nice to be able to

Here are some $\preceq$-versions.

Lemma 5.4 (Semi-Schanuel, onto $\preceq$-version). Given homomorphisms $\mathcal{P}_{1} \stackrel{f_{1}}{\longrightarrow} \mathcal{M}$ and $\mathcal{P}_{2} \stackrel{f_{2}}{\longrightarrow} \mathcal{M}$ with $f_{2}$ onto,

(i) There is a submodule

$$
\mathcal{P}=\left\{\left(b_{1}, b_{2}\right): f_{1}\left(b_{1}\right)=f_{2}\left(b_{2}\right)\right\}
$$

of $\mathcal{P}_{1} \oplus \mathcal{P}_{2}$. Let $\pi_{i}$ denote the projection to $\mathcal{P}_{i}$ on the $i$-th coordinate, and $\pi_{i}^{\text {res }}$ is its restriction to $\mathcal{P}$. Then $\pi_{1}^{\text {res }}$ is onto.

(ii) There is a natural homomorphism $\pi: \operatorname{ker}_{N} \pi_{1}^{r e s} \rightarrow \mathcal{P}_{2}$ via

$$
\left(\left(b_{1}, b_{2}\right),\left(b_{1}, b_{2}^{\prime}\right)\right) \mapsto b_{2}(-) b_{2}^{\prime},
$$

which induces $a \preceq$-morphism

$$
\operatorname{ker}_{N} \pi_{1}^{\mathrm{res}} \rightarrow \operatorname{ker}_{\mathrm{Mod}, \mathcal{P}_{2}} f_{2} \text {. }
$$

(iii) $\operatorname{ker}_{\text {Mod, } \mathcal{P}} \pi_{1}^{\text {res }}=\left\{\left(b_{1}, b_{2}\right) \in \mathcal{P}: b_{1} \succeq \mathbb{O}, b_{2} \in \operatorname{ker}_{\text {Mod, } \mathcal{P}} f_{2}\right\}$.

(iv) $f_{1} \pi_{1}^{\text {res }}\left(b_{1}, b_{2}\right)=f_{2} \pi_{2}^{r e s}\left(b_{1}, b_{2}\right)$.

(v) If $\mathcal{P}_{1}$ is h-projective, then it is a h-retract of $\mathcal{P}$ with respect to the projection $\pi_{1}: \mathcal{P} \rightarrow \mathcal{P}_{1}$, and $\mathcal{P}$ is the $h$-direct sum of $\mathcal{P}_{1}$ and $\left(\mathbb{1}_{\mathcal{P}}(-) \nu_{1} \pi_{1}\right)(\mathcal{P})$. 
Proof. (i) The same argument in Theorem 5.3 works.

(ii) As in Theorem 5.3(i), we have that

$$
\operatorname{ker}_{N} \pi_{1}^{\text {res }} \subseteq\left\{\left(\left(b_{1}, b_{2}\right),\left(b_{1}^{\prime}, b_{2}^{\prime}\right)\right): b_{1}=b_{1}^{\prime}\right\} .
$$

But, by the definition of $\pi_{1}^{\text {res }}$ and $\mathcal{P}$, we see, for $\left(\left(b_{1}, b_{2}\right),\left(b_{1}, b_{2}^{\prime}\right)\right) \in \operatorname{ker}_{N} \pi_{1}^{\text {res }}$, that

$$
f_{1}\left(b_{1}\right)=f_{2}\left(b_{2}\right)=f_{2}\left(b_{2}^{\prime}\right) \text {, }
$$

and hence

$$
\operatorname{ker}_{N} \pi_{1}^{r e s}=\left\{\left(\left(b_{1}, b_{2}\right),\left(b_{1}, b_{2}^{\prime}\right)\right): b_{1} \in \mathcal{P}_{1}, f_{2}\left(b_{2}\right)=f_{2}\left(b_{2}^{\prime}\right)\right\} .
$$

Therefore, we obtain

$$
f_{2}\left(b_{2}\right)(-) f_{2}\left(b_{2}^{\prime}\right)=f_{2}\left(b_{2}(-) b_{2}^{\prime}\right) \succeq \mathbb{0} .
$$

Hence $\pi\left(\operatorname{ker}_{N} \pi_{1}^{r e s}\right) \subseteq \operatorname{ker}_{\text {Mod, }, \mathcal{P}_{2}} f_{2}$.

(iii) $(\supseteq)$ is clear. Conversely, suppose $\pi_{1}^{\text {res }}\left(b_{1}, b_{2}\right) \succeq \mathbb{0}$. Then $b_{1} \succeq \mathbb{0}$, implying $f_{2}\left(b_{2}\right)=f_{1}\left(b_{1}\right) \succeq \mathbb{0}$, i.e., $b_{2} \in \operatorname{ker}_{\text {Mod, } \mathcal{P}} f_{2}$.

(iv) $f_{1} \pi_{1}^{\text {res }}\left(b_{1}, b_{2}\right)=f_{1}\left(b_{1}\right)=f_{2}\left(b_{2}\right)=f_{2} \pi_{2}^{r e s}\left(b_{1}, b_{2}\right)$.

(v) Take $\nu_{1}: \mathcal{P}_{1} \rightarrow \mathcal{P}$ be the homomorphism $h$-splitting $\pi_{1}$ via the identity map on $\mathcal{P}_{1}$, and we can apply Theorem 3.13 .

The next result, although not symmetric, does not require the onto hypothesis.

Lemma 5.5 (Semi-Schanuel, $\preceq$-onto $\preceq$-version). Take $\preceq=\preceq$ Null . Suppose we are given homomorphisms $\mathcal{P}_{1} \stackrel{f_{1}}{\longrightarrow} \mathcal{M}$ and $\mathcal{P}_{2} \stackrel{f_{2}}{\longrightarrow} \mathcal{M}$ with $f_{2} \preceq$-onto.

(i) There is a submodule

$$
\mathcal{P}_{\preceq}=\left\{\left(b_{1}, b_{2}\right): f_{1}\left(b_{1}\right) \preceq f_{2}\left(b_{2}\right)\right\}
$$

of $\mathcal{P}_{1} \oplus \mathcal{P}_{2}$. Let $\pi_{i}$ denote the projection to $\mathcal{P}_{i}$ on the $i$-th coordinate, and $\pi_{i}^{\text {res }}$ is its restriction to $\mathcal{P} \prec$. Then $\pi_{1}^{\text {res }}$ is onto.

(ii) There is a natural homomorphism $\operatorname{ker}_{N} \pi_{1}^{r e s} \rightarrow \mathcal{P}_{2}$ via

$$
\left(\left(b_{1}, b_{2}\right),\left(b_{1}, b_{2}^{\prime}\right)\right) \mapsto b_{2}(-) b_{2}^{\prime},
$$

which restricts to a homomorphism $\pi: \operatorname{ker}_{N, \preceq} \pi_{1}^{r e s} \rightarrow \mathcal{P}_{2}$ whose image is a subset of the following set

$$
\left\{b_{2}(-) b_{2}^{\prime}: f\left(b_{2}\right)(-) f\left(b_{2}^{\prime}\right) \in \mathcal{M}^{\circ}\right\} .
$$

Furthermore, we have $\pi: \operatorname{ker}_{N}, \pi_{1}^{\mathrm{res}} \rightarrow \operatorname{ker}_{\mathrm{Mod}, \mathcal{P}_{2}} f_{2}$.

(iii) $\operatorname{ker}_{\mathrm{Mod}, \mathcal{P} \preceq} \pi_{1}^{\text {res }}=\left\{\left(b_{1}, b_{2}\right) \in \mathcal{P}_{\preceq}: b_{1} \succeq \mathbb{O}, b_{2} \in \operatorname{ker}_{\mathrm{Mod}, \mathcal{P}} f_{2}\right\}$.

(iv) $f_{1} \pi_{1}^{\text {res }}\left(b_{1}, b_{2}\right) \preceq f_{2} \pi_{2}^{r e s}\left(b_{1}, b_{2}\right)$.

Proof. (i) The proof is similar to the previous cases. Clearly $\mathcal{P}_{\preceq}$ is a submodule of $\mathcal{P}_{1} \oplus \mathcal{P}_{2}$. Since $f_{2}$

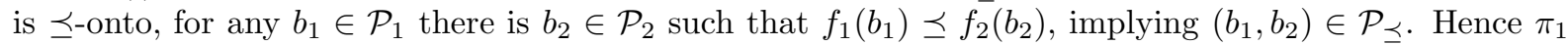
restricts to an onto homomorphism $\pi_{1}^{\text {res }}: \mathcal{P}_{\preceq} \rightarrow \mathcal{P}_{1}$.

(ii) As before, we have that

$$
\operatorname{ker}_{N} \pi_{1}^{\text {res }} \subseteq\left\{\left(\left(b_{1}, b_{2}\right),\left(b_{1}^{\prime}, b_{2}^{\prime}\right)\right): b_{1}=b_{1}^{\prime}\right\} .
$$

Hence, the map

$$
\operatorname{ker}_{N} \pi_{1}^{r e s} \rightarrow \mathcal{P}_{2}, \quad\left(\left(b_{1}, b_{2}\right),\left(b_{1}, b_{2}^{\prime}\right)\right) \mapsto b_{2}(-) b_{2}^{\prime}
$$

is well-defined and clearly a homomorphism. Furthermore, we can restrict (5.1) to a homomorphism

$$
\pi: \operatorname{ker}_{N, \preceq} \pi_{1}^{r e s} \rightarrow \mathcal{P}_{2}, \quad\left(\left(b_{1}, b_{2}\right),\left(b_{1}, b_{2}^{\prime}\right)\right) \mapsto b_{2}(-) b_{2}^{\prime}
$$

since $\operatorname{ker}_{N, \preceq} \pi_{1}^{r e s} \subseteq \operatorname{ker}_{N} \pi_{1}^{r e s}$. Now, let

$$
X:=\left\{b_{2}(-) b_{2}^{\prime}: f\left(b_{2}\right)(-) f\left(b_{2}^{\prime}\right) \in \mathcal{M}^{\circ}\right\} .
$$

For $\alpha:=\left(\left(b_{1}, b_{2}\right),\left(b_{1}, b_{2}^{\prime}\right)\right) \in \mathcal{P}_{\preceq} \times \mathcal{P}_{\preceq}$, it is clear that $\alpha \in \operatorname{ker}_{N, \preceq} \pi_{1}^{r e s}$ if and only if $b_{1} \succeq \mathbb{O}$. Furthermore, as $\alpha \in \mathcal{P}_{\preceq} \times \mathcal{P}_{\preceq}$, in this case, we have that

$$
\mathbb{0} \preceq f_{1}\left(b_{1}\right) \preceq f_{2}\left(b_{2}\right), \quad \mathbb{0} \preceq f_{1}\left(b_{1}\right) \preceq f_{2}\left(b_{2}^{\prime}\right) .
$$


So writing $f_{2}\left(b_{2}\right)=f_{1}\left(b_{1}\right)+c$ and $f_{2}\left(b_{2}^{\prime}\right)=f_{1}\left(b_{1}\right)+c^{\prime}$ for some $c, c^{\prime} \succeq \mathbb{0}$, we obtain

$$
f_{2}\left(b_{2}\right)(-) f_{2}\left(b_{2}^{\prime}\right)=f_{1}\left(b_{1}\right)^{\circ}+c(-) c^{\prime} \succeq \mathbb{0},
$$

in particular, $f_{2}\left(b_{2}\right)(-) f_{2}\left(b_{2}^{\prime}\right) \in \mathcal{M}^{\circ}$. Therefore, we have that $\pi(\alpha) \in X$.

Since $f_{2}$ is a homomorphism, we have that $f\left(b_{2}\right)(-) f\left(b_{2}^{\prime}\right)=f_{2}\left(b_{2}(-) b_{2}^{\prime}\right)$ and hence the set $X$ becomes the following set

$$
X=\left\{b_{2}(-) b_{2}^{\prime}: f_{2}\left(b_{2}(-) b_{2}^{\prime}\right) \in \mathcal{M}^{\circ}\right\},
$$

in particular, $X \subseteq \operatorname{ker}_{\text {Mod, }} \mathcal{P}_{2} f_{2}$ and hence we have $\pi: \operatorname{ker}_{N, \preceq} \pi_{1}^{\text {res }} \rightarrow \operatorname{ker}_{\text {Mod, } \mathcal{P}_{2}} f_{2}$.

(iii) $(\supseteq)$ is clear. Conversely, suppose $\pi_{1}^{\text {res }}\left(b_{1}, b_{2}\right) \succeq \mathbb{0}$. Then $b_{1} \succeq \mathbb{0}$, implying $f_{2}\left(b_{2}\right) \succeq f_{1}\left(b_{1}\right) \succeq \mathbb{0}$, i.e., $b_{2} \in \operatorname{ker}_{\text {Mod, }, \mathcal{P}} f_{2}$.

(iv) $f_{1} \pi_{1}^{\text {res }}\left(b_{1}, b_{2}\right)=f_{1}\left(b_{1}\right) \preceq f_{2}\left(b_{2}\right)=f_{2} \pi_{2}^{r e s}\left(b_{1}, b_{2}\right)$.

We also have the following $\preceq$ analogs of the classical proof of Schanuel.

Theorem 5.6 (Semi-Schanuel, another $\preceq$-version). Given $a \preceq$-morphism $\mathcal{P} \stackrel{f}{\longrightarrow} \mathcal{M}$ and a homomorphism $\mathcal{P}^{\prime} \stackrel{f^{\prime}}{\longrightarrow} \mathcal{M}^{\prime}$ with $\mathcal{P}$ and $\mathcal{P}^{\prime} \preceq$-projective and $f \preceq$-onto, and a $\preceq$-onto $\preceq$-morphism $\mu: \mathcal{M} \rightarrow \mathcal{M}^{\prime}$, let $\mathcal{K}=\operatorname{ker}_{\text {Mod, } \mathcal{P}} f$ and $\mathcal{K}^{\prime}=\operatorname{ker}_{\text {Mod, }} \mathcal{P}^{\prime} f^{\prime}$. Then there is a $\preceq$-onto $\preceq$-splitting $\preceq$-morphism $g: \mathcal{K}^{\prime} \oplus \mathcal{P} \rightarrow \mathcal{P}^{\prime}$, with a $\preceq$-morphism $\Phi: \mathcal{K} \rightarrow \operatorname{ker}_{\mathrm{Mod}, \mathcal{K}^{\prime} \oplus \mathcal{P}} g$ which is 1:1 (as a set-map).

Proof. Lifting $\mu f$ to a $\preceq$-morphism $h: \mathcal{P} \rightarrow \mathcal{P}^{\prime}$ satisfying $\mu f \preceq f^{\prime} h$, define $g: \mathcal{K}^{\prime} \oplus \mathcal{P} \rightarrow \mathcal{P}^{\prime}$ by $g\left(b^{\prime}, b\right)=h(b)(-) b^{\prime}$.

We first claim that $g$ is $\preceq$-onto. In fact, one may observe that for any $b^{\prime} \in \mathcal{P}^{\prime}$, there exists $b \in \mathcal{P}$ such that

$$
\mu f(b) \succeq f^{\prime}\left(b^{\prime}\right) .
$$

Indeed, let $c=f^{\prime}\left(b^{\prime}\right)$. Since $\mu$ is $\preceq$-onto, there exists $x \in \mathcal{M}$ such that $c \preceq \mu(x)$. Moreover, since $f$ is $\preceq$-onto, we have $b \in \mathcal{P}$ such that $x \preceq f(b)$, in particular, we have that

$$
f^{\prime}\left(b^{\prime}\right)=c \preceq \mu(x) \preceq \mu f(b) .
$$

Since $f^{\prime}$ is a homomorphism, this implies that

$$
f^{\prime}\left(h(b)(-) b^{\prime}\right)=f^{\prime}(h(b))(-) f^{\prime}\left(b^{\prime}\right) \succeq \mu f(b)(-) f^{\prime}\left(b^{\prime}\right) \succeq 0 .
$$

Therefore, we have that

$$
h(b)(-) b^{\prime} \in \mathcal{K}^{\prime} .
$$

Furthermore, we have that

$$
g\left(h(b)(-) b^{\prime}, b\right)=h(b)(-)\left(h(b)(-) b^{\prime}\right) \succeq b^{\prime},
$$

implying $g$ is $\preceq$-onto. Since $\mathcal{P}^{\prime}$ is $\preceq$-projective, $g \preceq$-splits (see, Remark 4.5.)

For the last assertion that there is a $\preceq$-monic $\Phi: \mathcal{K} \rightarrow \operatorname{ker}_{\text {Mod, } \mathcal{K}^{\prime} \oplus \mathcal{P}} g$, take the map

$$
\Phi: \mathcal{K} \rightarrow \operatorname{ker}_{\mathrm{Mod}, \mathcal{K}^{\prime} \oplus \mathcal{P}} g, \quad b \mapsto(h(b), b) .
$$

One can easily see that $\Phi$ is well-defined since $b \in \mathcal{K}$ implies that

$$
f^{\prime} h(b) \succeq \mu f(b) \succeq \mu(\mathbb{O})=\mathbb{O},
$$

showing that $h(b) \in \mathcal{K}^{\prime}$, also

$$
g(h(b), b)=h(b)(-) h(b) \succeq \mathbb{0},
$$

showing that $(h(b), b) \in \operatorname{ker}_{\text {Mod, } \mathcal{K}^{\prime} \oplus \mathcal{P}} g$. Finally, it is clear that $\Phi$ is one-to-one as a set-map.

Theorem 5.6 can sometimes be used in conjunction with Theorem 4.15

Corollary 5.7. In the notation of Theorem 5.6, if $\mathcal{K}$ is $\preceq$-projective and the map $\Phi$ is the $\preceq$-retract of a split $\preceq$-morphism $\operatorname{ker}_{\mathrm{Mod}, \mathcal{K}^{\prime} \oplus \mathcal{P}} \rightarrow \mathcal{K}$, then $\mathcal{K}^{\prime}$ also is $\preceq$-projective.

Proof. $\operatorname{ker}_{\text {Mod, } \mathcal{K}^{\prime}} g$ is $\preceq$-projective, implying $\mathcal{K}^{\prime} \oplus \mathcal{P}$ is $\preceq$-projective, and thus $\mathcal{K}^{\prime}$ is $\preceq$-projective by Lemma 4.8 


\section{REFERENCES}

[1] M. Akian, S. Gaubert, and Rowen, L., Linear algebra over systems, Preprint (2019).

[2] L. Anderson and J. Davis, Hyperfield Grassmannians, Advances in Mathematics, 341 (2019), 336-366.

[3] M. Baker and N. Bowler, Matroids over partial hyperstructures, Advances in Mathematics 343 (2019): 821-863.

[4] M. Baker and O. Lorscheid, The moduli space of matroids, arXiv preprint arXiv:1809.03542.

[5] M. Baker and O. Lorscheid, Descartes' rule of signs, Newton polygons, and polynomials over hyperfields, arXiv preprint arXiv:1811.04966

[6] G. Cohen, S. Gaubert, and J.P Quadrat, Linear projectors in max-plus algebra. Proceedings of the IEEE-Mediterranean Conference, Cyprus, July 1997.

[7] A. Connes and C. Consani, The hyperring of adèle classes. J. Number Theory, 131(2) (2011), 159-194.

[8] A. Connes and C. Consani, Homological algebra in characteristic one, arXiv preprint arXiv:1703.02325

[9] A.A. Costa. Sur la théorie générale des demi-anneaux, Publ. Math. Debrecen 10:14-29, 1963.

[10] R.P. Deore and K.B. Pati, On the dual basis of projective semimodules and its applications, Sarajevo Journal of Mathematics 1 (14) (2005), 161-169.

[11] A. Dress, Duality theory for finite and infinite matroids with coefficients, Advances in Mathematics 93(2) (1986), 214-250.

[12] J. Flores, Homological algebra for commutative monoids, arXiv:1503.02309 (2015).

[13] S. Gaubert, Two lectures on max-plus algebra, Proceedings of the 26th Spring School of Theoretical Computer Science, Algèbres Max-Plus et applications en informatique et automatique", Ile de Noirmoutier, 4-7 mai, INRIA, 83-147, 1998.

[14] L. Gatto and L. Rowen, Grassman semialgebras and the Cayley-Hamilton theorem, arXiv:1803.08093.

[15] S. Gaubert, Théorie des systèmes linéaires dans les diodes. Thèse, École des Mines de Paris, 1992.

[16] J. Giansiracusa, J. Jun, and O. Lorscheid, On the relation between hyperrings and fuzzy rings, Beitr. Algebra Geom. DOI 10.1007/s13366-017-0347-5, 2017.

[17] J. Golan, Semirings and their Applications, Springer-Science + Business, Dordrecht, 1999. (Previously published by Kluwer Acad. Publ., 1999.)

[18] Z. Izhakian, Tropical arithmetic and matrix algebra, Commun. in Algebra, 37(4):1445-1468, 2009.

[19] Z. Izhakian, M. Johnson, and M. Kambites, Pure dimension and projectivity of tropical polytopes, Advances in Mathematics 303 (2016), 1236-1263.

[20] Z. Izhakian, M. Knebusch, and L. Rowen, Decompositions of Modules lacking zero sums, arXiv:1511.04041, Israel J. Math, to appear.

[21] Z. Izhakian, M. Knebusch, and L. Rowen, Summand absorbing submodules of a module over a semiring, arXiv:1705.10089, JPAA, to appear (2019).

[22] Z. Izhakian and L. Rowen, Supertropical algebra, Advances in Mathematics, 225(4)(2010), 2222-2286.

[23] D. Joo and K. Mincheva, Prime congruences of idempotent semirings and a Nullstellensatz for tropical polynomials, Selecta Mathematica doi:10.1007/s00029-017-0322-x (2017).

[24] J. Jun, Geometry of hyperfields, arXiv preprint arXiv:1707.09348 (2017).

[25] J. Jun, K. Mincheva, and L. Rowen, Homology of module systems, in preparation.

[26] J. Jun and L. Rowen, Categories with negation, arXiv preprint arXiv 1709.0318.

[27] Y. Katsov, Tensor products of functors, Siberian J. Math. 19 (1978), 222-229, trans. from Sirbiskii Mathematischekii Zhurnal 19 no. 2 (1978), 318-327.

[28] Y. Katsov, Toward homological characterization of semirings: Serre's conjecture and Bass' perfectness in a semiring context, Algebra Universalis 52 (2004), 197-214.

[29] Y. Katsov and T.G. Nam, Morita equivalence and homological characterization of semirings, Journal of Algebra and Its Applications Vol. 10, No. 3 (2011), 445-473.

[30] Y. Katsov, T.G. Nam, and J. Zumbrägel, On congruence-semisimple semirings and the $k_{0}$-group characterization of ultramatricial algebras over semifields, arXiv preprint arXiv:1711.05163.

[31] O. Lorscheid, The geometry of blueprints Part I, Advances in Mathematics 229, no. 3, 1804-1846, 2012.

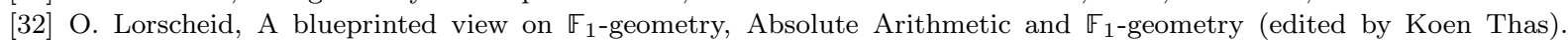
European Mathematical Society Publishing House, 2016.

[33] T.Y. Lam, Ring Theory, Lectures on Modules and Rings. Springer (1999).

[34] D. Maclagan and B. Sturmfels, Introduction to tropical geometry, American Mathematical Society, 2015.

[35] A.W. Macpherson, Projective modules over polyhedral semirings arXiv preprint arXiv:1507.07213

[36] G. Mikhalkin, I. Zharkov, Tropical curves, their Jacobians and theta functions, Curves and abelian varieties 465 (2008), 203-230.

[37] L Rowen, Ring Theory, Vol. I, Academic Press, Pure and Applied Mathematics 127, 1988.

[38] L. Rowen, Graduate algebra: Noncommutative view, AMS Graduate Studies in Mathematics 91 (2008).

[39] L. Rowen, Algebras with a negation map, arXiv:1602.00353

[40] L. Rowen, An informal overview of triples and systems, arXiv:1709.03174, NCRA V, Contemporary Mathematics Volume 727.

[41] L. Rowen, Morita theory of systems, arXiv: 1903.02412

[42] M. Takahashi, On the bordism categories III, Math. Seminar Notes Kobe University 10 (1982), 211-236.

[43] J. Trlifaj, Faith's problem on r-projectivity is undecidable, arXiv preprint arXiv:1710.10465 (2017). 
Department of Mathematics, University of Iowa, Iowa City, IA 52242, USA

E-mail address: jujun0915@gmail.com

Department of Mathematics, Yale University, New Haven, CT 06511, USA

E-mail address: kalina.mincheva@yale.edu

Department of Mathematics, Bar-Ilan University, Ramat-Gan 52900, Israel

E-mail address: rowen@math.biu.ac.il 